\title{
Toll-like receptor 4 (TLR4) deficiency aggravates dextran sulfate sodium (DSS)-induced intestinal injury by down-regulating IL6, CCL2 and CSF3
}

\author{
Yun-Jie Shi ${ }^{1 \#}$, Shi-Jie Hu ${ }^{2 \#}$, Quan-Quan Zhao ${ }^{1}$, Xiao-Shuang Liu ${ }^{1}$, Cong Liu ${ }^{3}$, Hao Wang ${ }^{1}$ \\ ${ }^{1}$ Department of Colorectal Surgery, Chang Hai Hospital, Naval Medical University (Second Military Medical University), Shanghai 200433, China; \\ ${ }^{2}$ Ningbo Anorectal Hospital, Ningbo 315104, China; ${ }^{3}$ Department of Radiation Medicine, Faculty of Naval Medicine, Naval Medical University \\ (Second Military Medical University), Shanghai 200433, China \\ Contributions: (I) Conception and design: H Wang, C Liu, YJ Shi; (II) Administrative support: H Wang; (III) Provision of study materials or patients: \\ C Liu; (IV) Collection and assembly of data: YJ Shi, QQ Zhao; (V) Data analysis and interpretation: YJ Shi, SJ Hu, XS Liu; (VI) Manuscript writing: \\ All authors; (VII) Final approval of manuscript: All authors. \\ \#These authors contributed equally to this work \\ Correspondence to: Hao Wang. Department of Colorectal Surgery, Chang Hai Hospital, Naval Medical University, Shanghai 200433, China. \\ Email: wanghaohh@vip.126.com; Cong Liu. Department of Radiation Medicine, Faculty of Naval Medicine, Naval Medical University, Shanghai \\ 200433, China. Email: victorliu20102020@163.com.
}

Background: Ulcerative colitis (UC) is an inflammatory bowel disease (IBD) that causes long-lasting inflammation and ulcers in the human digestive tract. The repair role of TLR4 in the intestinal epithelium is still unknown.

Methods: By comparing to wild-type (WT) mice, Toll-like receptor 4 (TLR4)-knockout mice (TLR4-KO) were used as dextran sulfate sodium (DSS)-induced colitis models to explore the role of TLR4 signaling in intestinal injury. High-throughput RNA-Seq, RT-qPCR and ELISA were performed to screen and verify key differences in gut genes between WT and TLR4-KO mice. Functional study of core dysregulated factors was performed in intestinal cell lines.

Results: We found that DSS-induced intestinal injury was aggravated by LPS (TLR4 agonist) and TLR4KO. When compared to WT mice, IL6, CCL2, CSF3, IL11, Ccnb1, Ccnd1 and TNF- $\alpha$ significantly decreased and Fas and FasL have increased in the gut of TLR4-KO mice. IL6, CCL2, CSF3, Fas and FasL have all increased in CT-26 cells treated with LPS. Combined with the above data and KEGG enrichment, it can be assumed that TLR4-KO might aggravate DSS-induced intestinal damage by attenuating cell cycle, cytokinecytokine receptor interaction, and Toll-like receptor signaling pathway, and enhancing the apoptosis pathway. In the functional study of core dysregulated factors, it was found that LPS, IL6, IL11, CSF3, CCL2, S100A8, $S 100 A 9$ and $M m p 3$ have improved viability of colon cancer cell lines and decreased apoptosis rate of mouse colon cancer cells when these were treated with DSS. However, Jo-2 (Fas agonistic monoclonal antibody) played the opposite role in colon cancer cells treated with DSS.

Conclusions: TLR4 had a repairing effect on DSS-induced intestinal damage and it up-regulate IL6, CCL2 and CSF3. Fas and FasL enhanced DSS-induced colon injury in mice, but might have little to do with TLR4 signaling.

Keywords: Toll-like receptor 4 (TLR4); dextran sulfate sodium; IL6; CCL2; CSF3

Submitted Sep 30, 2019. Accepted for publication Nov 22, 2019.

doi: 10.21037/atm.2019.12.28

View this article at: http://dx.doi.org/10.21037/atm.2019.12.28 


\section{Introduction}

Inflammatory bowel disease (IBD) is categorized into ulcerative colitis (UC) and Crohn's disease (CD). It seriously disturbs the normal function of the digestive tract (1). IBD pathogenesis is still not clear. Some reports suggest that gut microbiota act as initiators of intestinal inflammation (2). TLRs are sensors for pathogen-associated molecular patterns (PAMPs), and play important role in initiating inflammation (3). TLR4 is the main mediator of responses to lipopolysaccharide (LPS) in vitro and in vivo (4). TLR4/ $N F-\kappa B$ overactivation is believed to lead to uncontrolled immune response in the inflamed intestinal mucosa and to accelerate the progression of IBD (5). However, in our experimental system, TLR4 signaling might actually play a repairing role during the process of IBD, suggesting that the balancing role of TLR4 between intestinal injury and repair is important (6). Cario and colleagues (7) suggested that in the process of intestinal injury, intestinal stem cells at the base of the intestinal crypts repopulate the depleted surroundings, in a process known as compensatory proliferation. We hypothesized that the protective role of TLR4 signaling pathway in DSS-induced colitis is related to this compensatory proliferation response.

Our previous research showed that by upregulating $P G E_{2}$ and GM-CSF, TLR4 and its ligands might be contributing to epithelial repair after DSS-induced intestinal injury (6). However, at the time we did not conduct a detailed study on the specific downstream genes associated with TLR4 signaling and the alleviation DSS-induced colitis. In this study, we increased the number of WT and TLR4-knockout (TLR4-KO) mice to test the role of TLR4 in DSS-induced colitis. High-throughput RNA-Seq analysis was used to perform differential screen of key genes' expression in the guts of WT and TLR4-KO mice treated with DSS. Furthermore, we performed qRT-PCR and ELISA to confirm the expression of the genes identified by RNA-Seq analysis. Finally, we validated the key molecular functions using intestinal cell lines and, based on the results, built a key genes interactions network map.

\section{Methods}

\section{Mice}

Six-week-old wild-type (WT) mice (C57BL/6) weighing approximately $20 \mathrm{~g}$ each were purchased from the Chinese Academy of Science (Shanghai, China). Six-week-old TLR4KO mice (8) (C57BL/6, TLR4 gene deleted) weighing approximately $20 \mathrm{~g}$ each were provided by the Model Animal Research Center of Nanjing University (Nanjing, China). All mice were littermates and were maintained under specific pathogen-free (SPF) conditions in the Animal Center of Naval Medical University (Shanghai, China). Experimental groups were $n=5$ in intestinal injury evaluation experiments and $n=6$ in peripheral blood analysis experiments. Numbers of mice used in experimental groups in the survival studies are shown in the respective figures. RNA-Seq analysis was performed on intestinal samples from four pairs of WT and TLR4-KO mice. In verification experiments of quantitative real-time PCR (qRT-PCR) and enzyme-linked immunosorbent assay (ELISA), the number of mice per group was three. This study was approved by an institutional ethics committee and followed the tenets of the Declaration of Helsinki.

\section{Induction of DSS- induced colitis and LPS treatment}

For acute colitis model, mice were given different doses (\%, $\mathrm{g} / \mathrm{mL}$ ) of DSS (40,000 kDa; ICN Biochemicals, Irvine, CA, USA) in drinking water for seven days. Mice were switched to regular drinking water after that (9). Different doses of DSS were prepared based on the experimental purpose. The specific DSS doses used are indicated in each experimental figure. LPS from Escherichia coli was purchased from Sigma (St. Louis, MO, USA) and was re-purified. To explore the toxic effects of exogenous LPS, re-purified LPS was dissolved in pyrogen-free PBS (BaiSai, Shanghai, China). The WT mice were injected with LPS at doses of $0.1,0.5,2.5 \mathrm{mg} / \mathrm{kg}$ body weight for 3 days, and were then given the different doses $(\%, \mathrm{~g} / \mathrm{mL})$ of DSS. Control mice were injected with pyrogen-free PBS. In the experiments performed to study how TLR4 mediates the expression of $I L-6, C C L 2, C S F 3$, Fas, and FasL, CT-26 cells were treated with $100 \mathrm{ng} / \mathrm{mL}$ of LPS 24 hours before performing the measurements.

\section{Analysis of peripheral blood cells}

WT and TLR4-KO mice treated with $2.5 \%$ DSS were eyebled on day seven using heparin-coated capillary tubes to take blood while the mice were under anesthesia. The blood collected per mouse was $0.5-1.0 \mathrm{~mL}$. The collected blood was transferred to Eppendorf (EP) tubes with K2-EDTA and inverted multiple times. Peripheral blood index was determined by the standard five-classifications hematological analysis at the Animal Center of Naval Medical University. Peroximeter assay channels and basophil assay channels 
were used for leukocyte classification. White blood cells classification was performed according to the characteristics of cytochemically stained cells. A dual angle laser method was used in both red blood cells and platelets analyses.

\section{Fecal blood scoring}

Intestinal bleeding samples of WT and TLR4-KO mice treated with $2.5 \%$ DSS were dissected on day 7 . Colonic bleeding was determined via stool bleeding score (8) as follows: 0 , no gross blood visible anywhere through the entire colon; 1 , gross blood present in $<1 / 3$ of the colon; 2 , gross blood visible in $\geq 1 / 3$ but $<2 / 3$ of the colon; 3 , gross blood visible in $\geq 2 / 3$ of the colon.

\section{Pathological analysis}

Colon tissues of WT and TLR4-KO mice treated with $2.5 \%$ DSS were sampled on day seven of treatment. Colon tissue samples were fixed with $10 \%$ neutral formalin, paraffin embedded, sectioned at 2-7 $\mu \mathrm{m}$ thick and stained with haematoxylin and eosin (H\&E) (10). Photomicrographs of the desired sections were obtained using a digital research photographic microscope (Thermo Fisher Corporation, USA). Ki67 kits (Dakewe Biotech Corporation, Shenzhen, China) were applied to assess the proliferative capacity of the intestinal crypt epithelial cells (11).

\section{RNA-Seq and KEGG enrichment analyses}

Total RNA was extracted using the mirVana miRNA Isolation Kit (Ambion) following the manufacturer's protocol. RNA integrity was evaluated using the Agilent 2100 Bioanalyzer (Agilent Technologies, Santa Clara, CA, USA). Samples with RNA Integrity Number (RIN) $\geq 7$ were subjected to subsequent analysis. Libraries were constructed using TruSeq Stranded mRNA LTSample Prep Kit (Illumina, San Diego, CA, USA) according to the manufacturer's instructions. Libraries were then sequenced on the Illumina sequencing platform (HiSeqTM 2500 or Illumina HiSeq X Ten) and $125 \mathrm{bp} / 150 \mathrm{bp}$ pairedend reads were generated. A cut-off values of $\log 2$ (fold change) greater than 0.58 and $\mathrm{P}<0.05$ were used to extract the differentially expressed genes. Hierarchical cluster analysis (12) of differentially expressed transcript (DEGs) was performed to explore genes expression patterns. KEGG (13) pathway enrichment analysis of DEGs was performed using enrichR, based on the hypergeometric distribution. After differentially expressed genes were obtained, pathways with the number of differential genes greater than two were screened. From the total differential KEGG pathways between WT and TLR4-KO mice, we selected 15 signaling pathways that were closely related to DSS-induced colitis and performed a KEGG enrichment.

\section{Quantitative real-time PCR ( $q R T-P C R)$ and enzyme- linked immunosorbent assay (ELISA)}

Total RNA from tissue samples was isolated using RNA isolation and purification reagent kit provided by GenePharma Corporation (Shanghai, China). RNA concentration was measured using an ultraviolet spectrophotometer (GeneQuant, Pharmacia, USA), and was subsequently stored at $-80{ }^{\circ} \mathrm{C}$ pending analysis. All the primers were synthesized by Sangon Biotech Corporation (Shanghai, China). cDNA was synthesized using Hifair ${ }^{\circledR}$ II 1st Strand cDNA Synthesis Kit (YeaSen Biotech, Shanghai, China). Fluorescence qRT-PCR reactions were performed using SYBR Premix Ex Taq Kit (TaKaRa, Dalian, China) (14). Primers used for qRT-PCR are listed in Table 1.

To determine the level of IL6, CCL2 and CSF3 produced by the gut, each colon was divided into three segments of approximately $1 \mathrm{~cm}$ each. Colon segments were washed in phosphate-buffered saline (PBS) supplemented with penicillin and streptomycin (Gibco from Hyclone Corporation, United States). These segments were then cultured in 24-well flatbottom culture plates in serum-free 1640 medium (Gibco), supplemented with penicillin and streptomycin. After 24 hours, the supernatant fluid was collected and stored at $20^{\circ} \mathrm{C}$. Cytokines in the supernatant were measured using ELISA kits provided by Dakewe Biotech Corporation (Shenzhen, China) and were normalized for the number of cytokines per $\mathrm{mg}$ of total protein in the supernatant (8).

\section{Cell culture}

Mouse intestinal epithelial cell (IEC) line (CT-26) and human IEC line (SW1116) used in this study were obtained from the Shanghai Institute of Biochemistry and Cell Biology, Chinese Academy of Sciences (Shanghai, China). Cells were cultured in Dulbecco's modified Eagle's medium (DMEM; PAA, Austria) supplemented with 10\% fetal calf serum (PAA) at $37^{\circ} \mathrm{C}$ in a humidified atmosphere of $5 \% \mathrm{CO}_{2}$ in air. All cell lines used in this study were cultured for less than 6 months when the experiments were performed. 
Table 1 qRT-PCR primers for the 25 genes evaluated

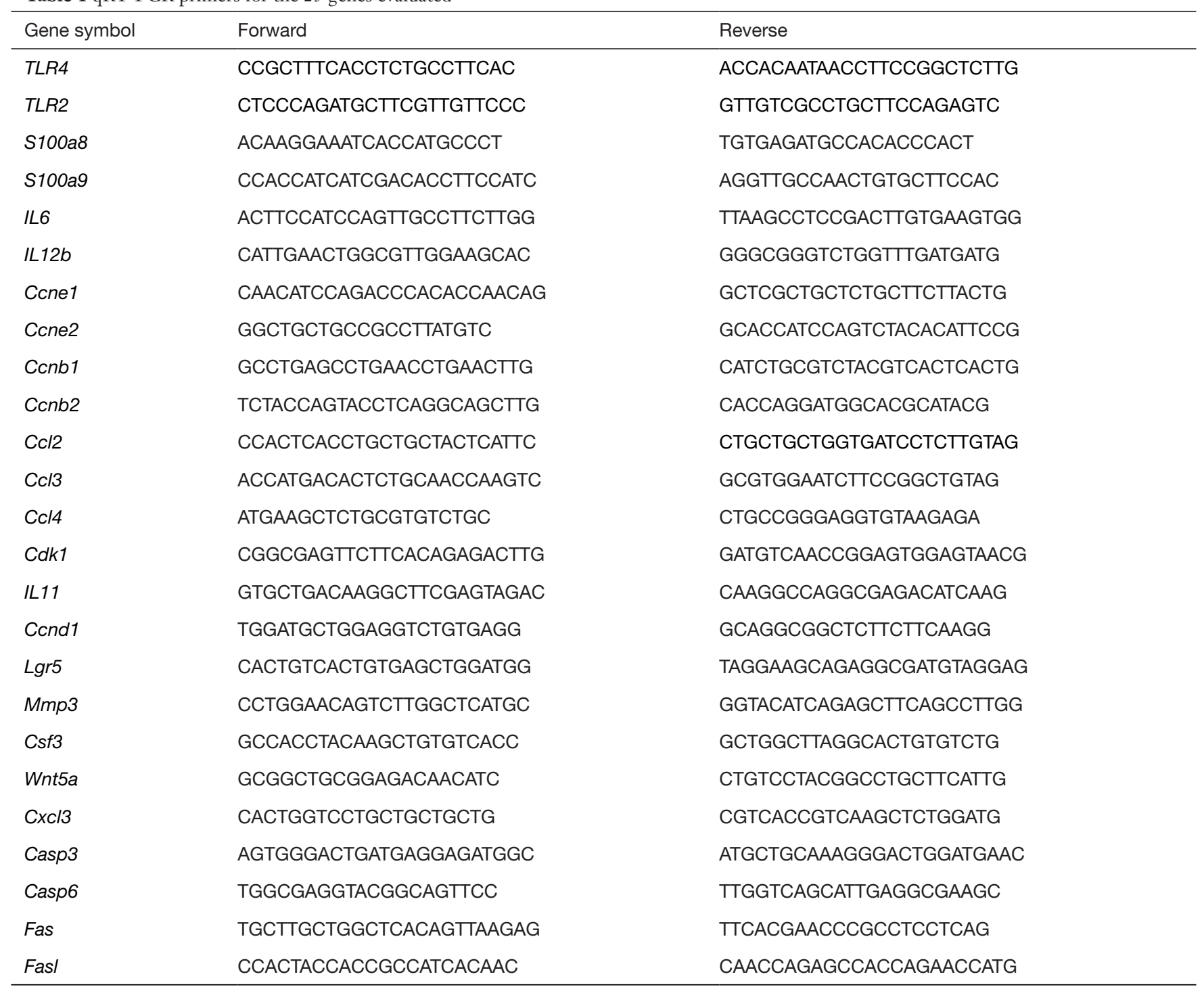

\section{MTT assay}

Relative cell viability was analyzed using tetrazolium salt 3-(4,5-dimethylthiazol-2-yl)-22,5-diphenyltetrazolium bromide (MTT) (Dojindo Corporation, Shanghai, China). CT-26 cells were treated with $100 \mathrm{ng} / \mathrm{mL}$ of LPS (Sigma Corporation, USA), IL6, IL11, CSF3, CCL2, S100A8/ A9, MMP3, Jo-2 (PeproTech Corporation, USA) for $24 \mathrm{~h}$. Cells were then treated with $0.5 \%$ DSS. After $24 \mathrm{~h}$, $20 \mu \mathrm{L}$ of MTT substrate were added to each well. The SW1116 cells were treated with $100 \mathrm{ng} / \mathrm{mL}$ of LPS (Sigma Corporation, USA), IL6, IL11, CCL2, S100A8, Jo-2 (PeproTech Corporation, USA) for $24 \mathrm{~h}$. Cells were then treated with $0.5 \%$ DSS. After $24 \mathrm{~h}, 20 \mu \mathrm{L}$ of
MTT substrate were added to each well. The plates were returned to the incubator for an additional $4 \mathrm{~h}$ at $37{ }^{\circ} \mathrm{C}$ and humidified atmosphere of $5 \% \mathrm{CO}_{2}$ in air. After the incubation stage, the medium was removed, the cells were solubilized in $150 \mu \mathrm{L}$ of dimethylsulfoxide and colorimetric analysis was then performed (wavelength: $490 \mathrm{~nm}$ ). The plates were analyzed on day 3 (15).

\section{Apoptosis analysis}

Cell apoptosis was analyzed using apoptosis detection kit (16) (TransGen Biotech, Beijing, China). CT-26 cells were treated with $100 \mathrm{ng} / \mathrm{mL}$ LPS (Sigma Corporation, 
A
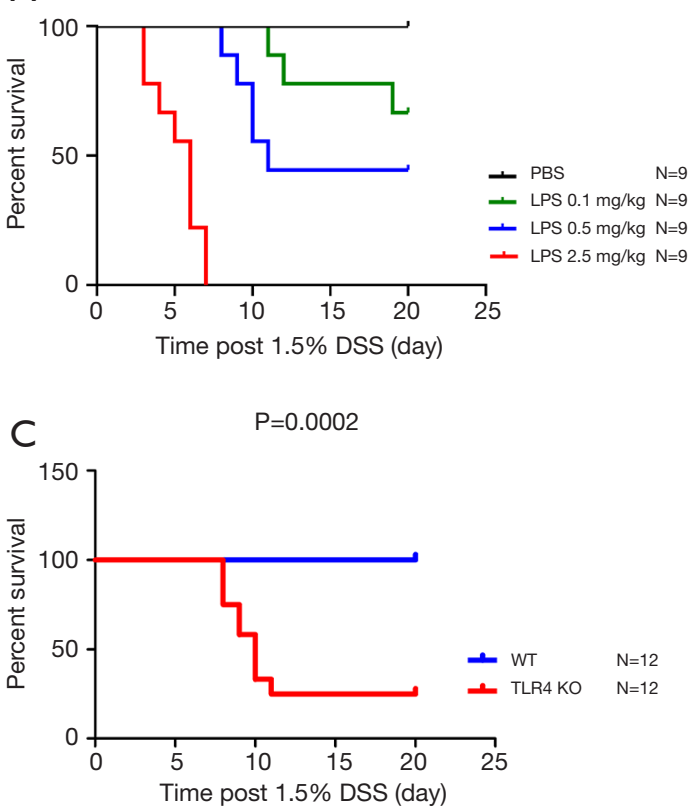

B
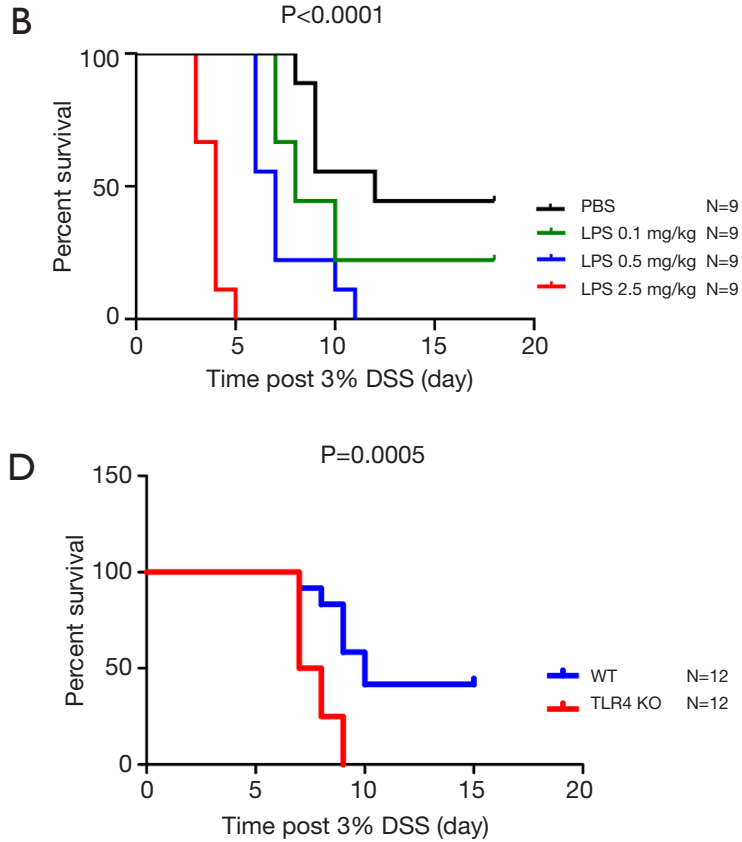

Figure 1 Exogenous LPS exacerbated dextran sulfate sodium (DSS)-induced colitis. Moreover, TLR4-KO mice displayed higher mortality compared to WT mice after receiving different doses of DSS. Survival rates were recorded for WT mice injected with PBS or different doses of LPS and, $24 \mathrm{~h}$ later, were given 1.5\% (A) or 3\% (B) DSS in the drinking water and for WT and TLR4-KO mice given 1.5\% (C) or 3\% (D) DSS in their drinking water.

USA), IL6, IL11, CSF3, CCL2, S100A8/A9, MMP3, Jo-2 (PeproTech Corporation, USA) for 24 hours, and were then exposed to $0.5 \%$ DSS. After $24 \mathrm{~h}$, the CT-26 cells were stained with Annexin V-FITC/propidium iodide (PI) (eBioscience Corporation, USA), and apoptosis rates were detected by flow cytometry (Beckman Coulter, USA).

\section{Transwell migration assay}

CT-26 cells were treated with $100 \mathrm{ng} / \mathrm{mL}$ LPS (Sigma Corporation, USA), IL6, IL11, CSF3, CCL2, S100A8, S100A9, Mmp3 and Jo-2 (PeproTech Corporation, USA) for $24 \mathrm{~h}$. The cells were then harvested and suspended in cell culture medium without FBS. The cells were seeded onto membranes in the absence of Matrigel. Complete medium containing FBS was added to the lower chamber, and non-migrated cells were gently removed with a cotton swab after 24 or $48 \mathrm{~h}$. Migratory cells located on the lower surface of the insert were fixed in $4 \%$ paraformaldehyde (Solarbio, Beijing, China), stained with $0.1 \%$ crystal violet (Sigma Corporation, USA), and counted for $53 \mathrm{~s}$ under flow cytometry (Beckman Coulter, USA) (17).

\section{Statistical analysis}

Statistical analysis was performed using the GraphPad Prism software (GraphPad Software Inc., La Jolla, CA, USA). All experiments were performed at least in triplicate. Comparisons between WT and TLR4-KO mice groups were performed using the Student's $t$-test. Differences in survival of the various mice groups were assessed using the Kaplan-Meier analysis and Cox regression analysis. The GraphPad software generated a $\mathrm{P}$ value and statistic for each analysis; $\mathrm{P}<0.05$ was considered statistically significant.

\section{Results}

\section{Exogenous LPS aggravated DSS-induced acute intestinal injury. Moreover, mice deficient in TLR4 showed even bigher susceptibility to DSS-induced intestinal injury}

The WT mice were injected with vehicle (PBS) or different doses of LPS $24 \mathrm{~h}$ before oral administration of $1.5 \%$ or $3 \%$ DSS. Animals that received LPS showed significantly higher mortality than did WT mice that were given PBS (Figure $1 A, B)$. These data suggest that LPS administration 
A

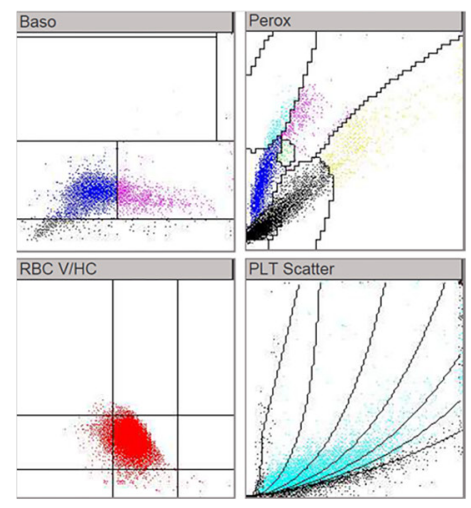

TLR4 KO

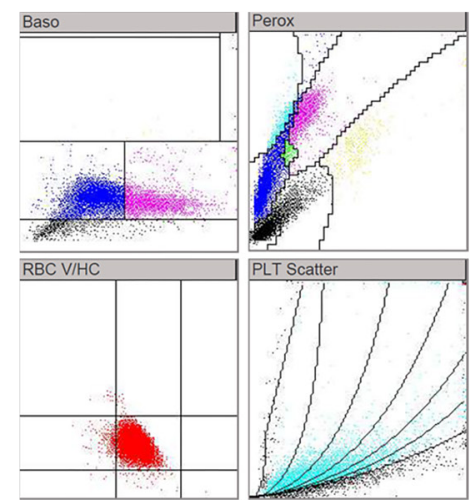

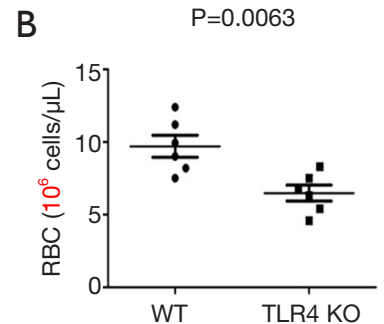

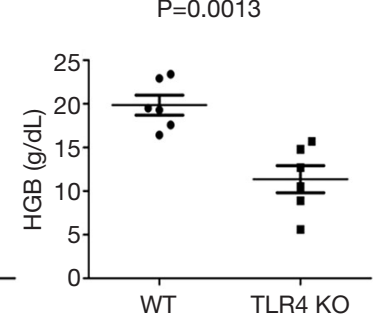

$\mathrm{P}=0.3426$
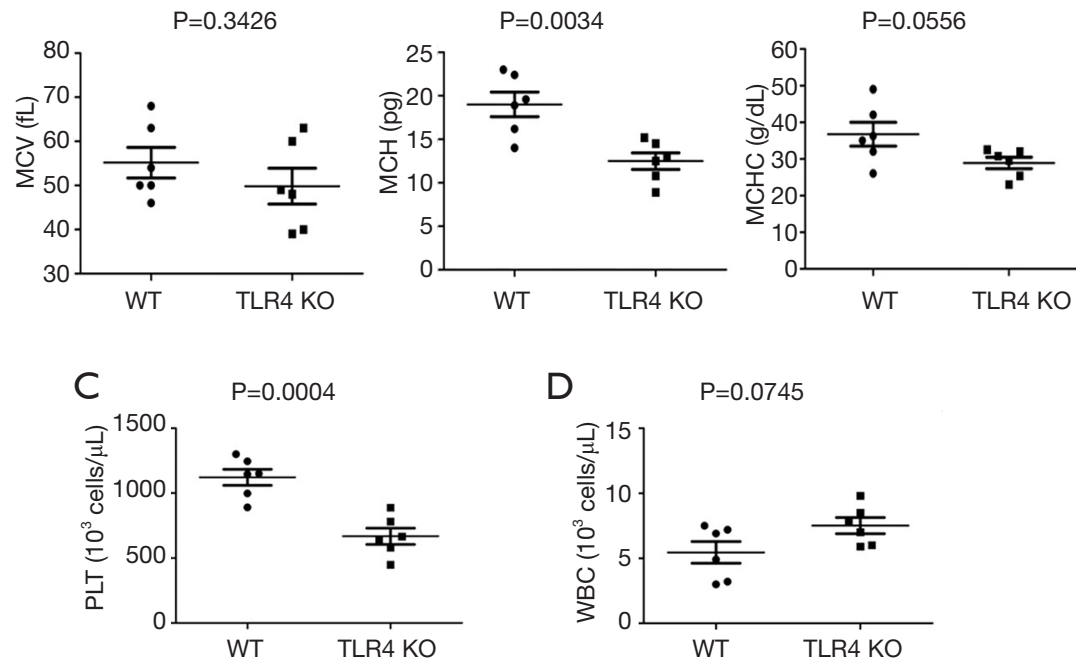

$\mathrm{P}=0.0025$
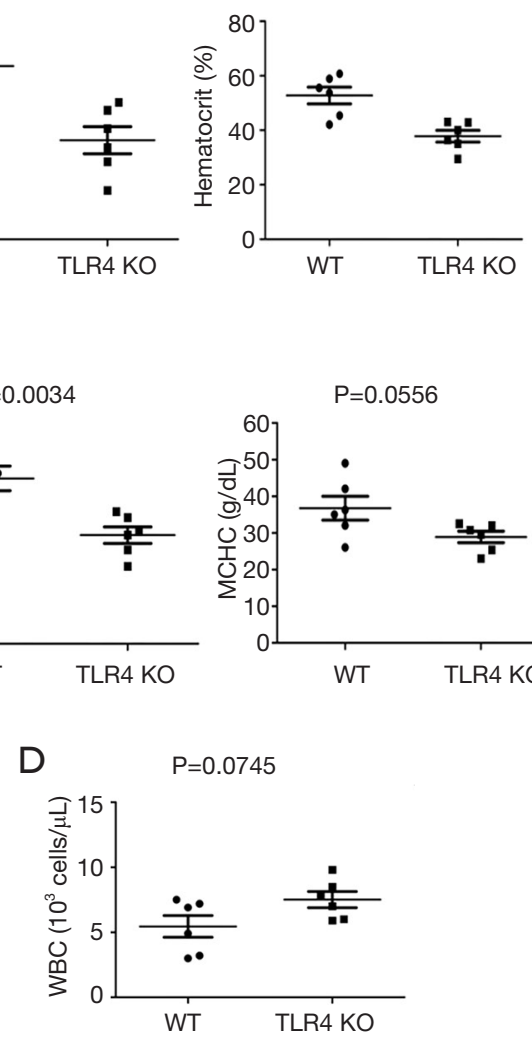

Figure 2 Mice deficient in TLR4 were more susceptible to anemia. (A) Representative peripheral blood cell analysis patterns of WT and TLR4-KO mice. In Baso pattern (top left), blue dots represent mononuclear cells, pink dots represent nucleated red blood cells or cell with lobed nuclei, the dots in the upper section of the quadrant represent basophilic granulocytes, and black dots represent primitive cells. In Perox pattern (top right), dark blue dots represent lymphocytes, green dots represent monocytes, pink dots represent neutrophil granulocytes, yellow dots represent eosinophilic granulocytes, and light blue dots represent unstained large cells. In the RBC V/HC pattern (bottom left), the $\mathrm{Y}$ axis denotes $\mathrm{RBC}$ volume and the $\mathrm{X}$ axis denotes hemoglobin concentration. In the PLT Scatter pattern (bottom right), the $\mathrm{Y}$ axis denotes platelet volume and the $\mathrm{X}$ axis denotes platelet content. Comparison of peripheral red blood cells (B), WBC (C), and platelets (D) in WT and TLR4-KO mice that received $2.5 \%$ dextran sulfate sodium (DSS) for 7 days.

aggravates DSS-induced acute intestinal injury. Yet, TLR4KO mice displayed more severe mortality than WT mice after receiving $1.5 \%$ or $3 \%$ DSS (Figure $1 C, D$ ). It is known that LPS can activate TLR4 signaling (18), so this result surprised us. We therefore analyzed the cause of death and morbidity of TLR4-KO mice, using several parameters. Measuring the peripheral blood index on day seven after receiving $2.5 \%$ DSS, TLR4-KO mice were more prone to anemia than WT mice (Figure $2 A$ ). They exhibited a significant decrease in red blood cell (RBC) count, hemoglobin (HGB) concentration, hematocrit, and platelet (PLT) count compared to WT mice (Figure 2B,C). However, white blood cells (WBC) displayed higher level trend in the peripheral blood of TLR4-KO mice, which may be related to the aggravated intestinal inflammation (Figure 2D). Consistent with symptoms of anemia, TLR4-KO mice intestinal bleeding was more serious than in WT mice after ingesting $2.5 \%$ DSS for 7 days (Figure 3A,B). Histological study, performed at the end of the 7-day treatment with $2.5 \%$ DSS, indicated that extensive superficial ulceration with severe acute inflammation in TLR4-KO mice colon was more severe than that in the colon of WT mice. The colon of TLR4-KO mice also displayed extensive surface epithelium denudation (Figure 3C). Immunohistological Ki67 assay showed that, compared to WT mice, the proliferation of IEC in TLR4-KO mice 
A

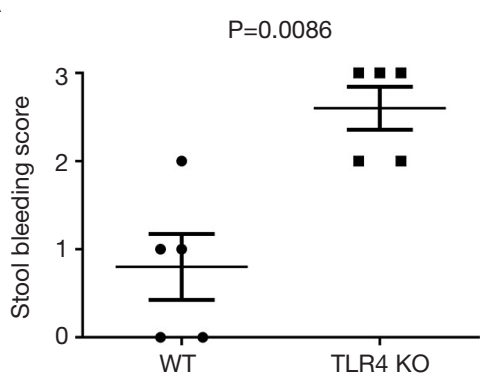

B

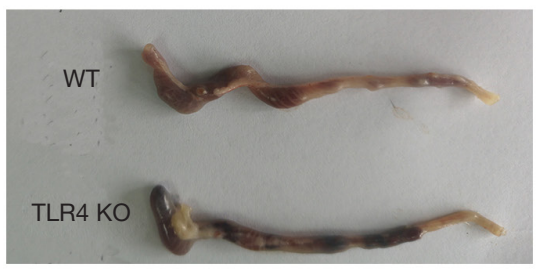

\section{$\mathrm{E}$}

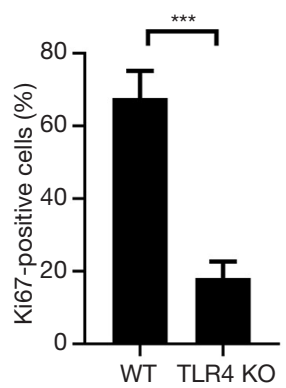

C
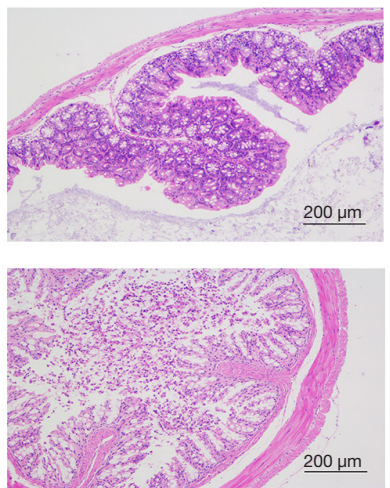

TLR4 KO
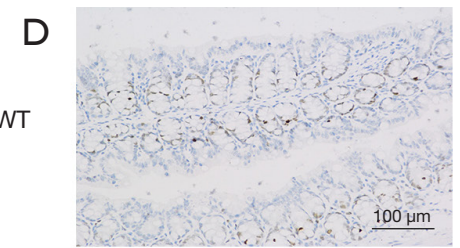

WT

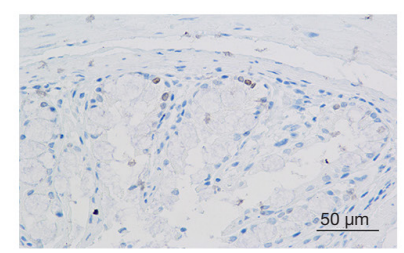

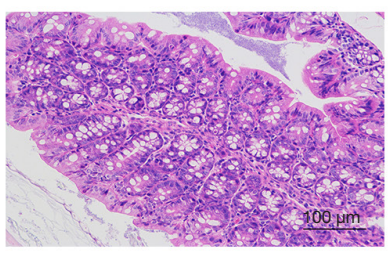
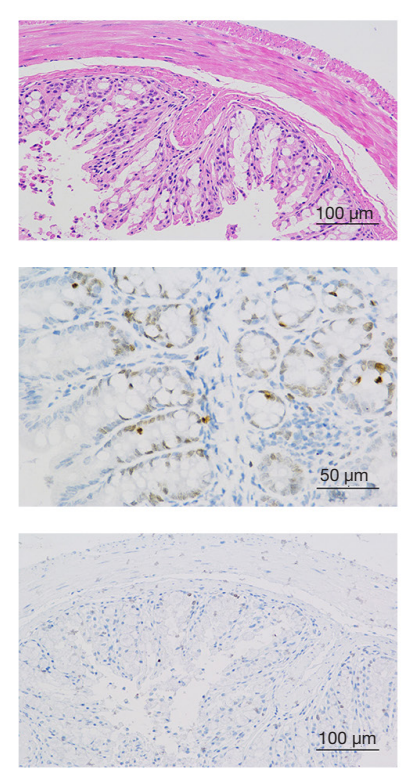

Figure 3 Compared with WT mice, the intestinal damage of TLR4-KO mice was more severe. (A) Stool bleeding score: 0 , no gross blood visible throughout the entire colon; 1 , gross blood present in $<1 / 3$ of the colon; 2 , gross blood visible in $>1 / 3$ but $<2 / 3$ of the colon; 3 , gross blood visible in $\geq 2 / 3$ of the colon. (B) Image of representative colons from WT and TLR4-KO mice after 7 days of treatment with $2.5 \%$ dextran sulfate sodium (DSS). (C) H\&E-stained images (magnification, $\times 100, \times 200$ ) of colons from WT and TLR4-KO mice after 7 days of treatment with 2.5\% DSS treatment. (D) Immunohistological Ki67 assay images (magnification, $\times 200, \times 400$ ) of colons from WT and TLR4KO mice after receiving 2.5\% DSS treatment for 7 days. (E) Comparison between WT and TLR4-KO mice based on Ki67-positive cells in intestines. The comparisons were performed after treating the mice with $2.5 \%$ DSS for 7 days. ${ }^{* *}, \mathrm{P}<0.001$.

was significantly weakened after oral administration of $2.5 \%$ DSS (Figure 3D,E).

\section{Differential genes expression patterns analysis in guts from WT and TLR4-KO mice after DSS treatment}

To determine how TLR4 exerts a protective effect in DSSinduced colitis, we compared the global gene expression profiles in the gut between WT and TLR4-KO mice. A cut-off value of a $\log 2$ (fold change) greater than 0.58 and $\mathrm{P}<0.05$ were used to detect the differentially expressed genes. A total of 7,273 differential genes were detected by RNA-Seq in 4 pairs of colon samples from WT and TLR4KO mice. Hierarchal clustering analysis indicated that the gene expression patterns in the colon were distinguishable between WT and TLR4-KO mice after DSS treatment (Figure 4A). Compared with WT mice, there were 3,503 significantly up-regulated and 3,769 significantly downregulated genes in guts of TLR4-KO mice. Volcano plot helped visualize the significantly different up-regulated and down-regulated genes in the guts of TLR4-KO mice (Figure $4 B$ ). We selected 54 genes that we pinpointed based on our RNA-Seq data sets, such as IL6, CSF3, CCL2, Fas and Fas-L, and created a heat map of their expression (Figure $4 C$ ). These 54 genes are associated with inflammatory responses and TLR4 signaling. We are familiar with these genes as we frequently use them in our experimental system. As expected, the selected genes expression patterns in the colons were significantly distinguishable between the WT and TLR4-KO mice after DSS treatment (Figure 4C). 

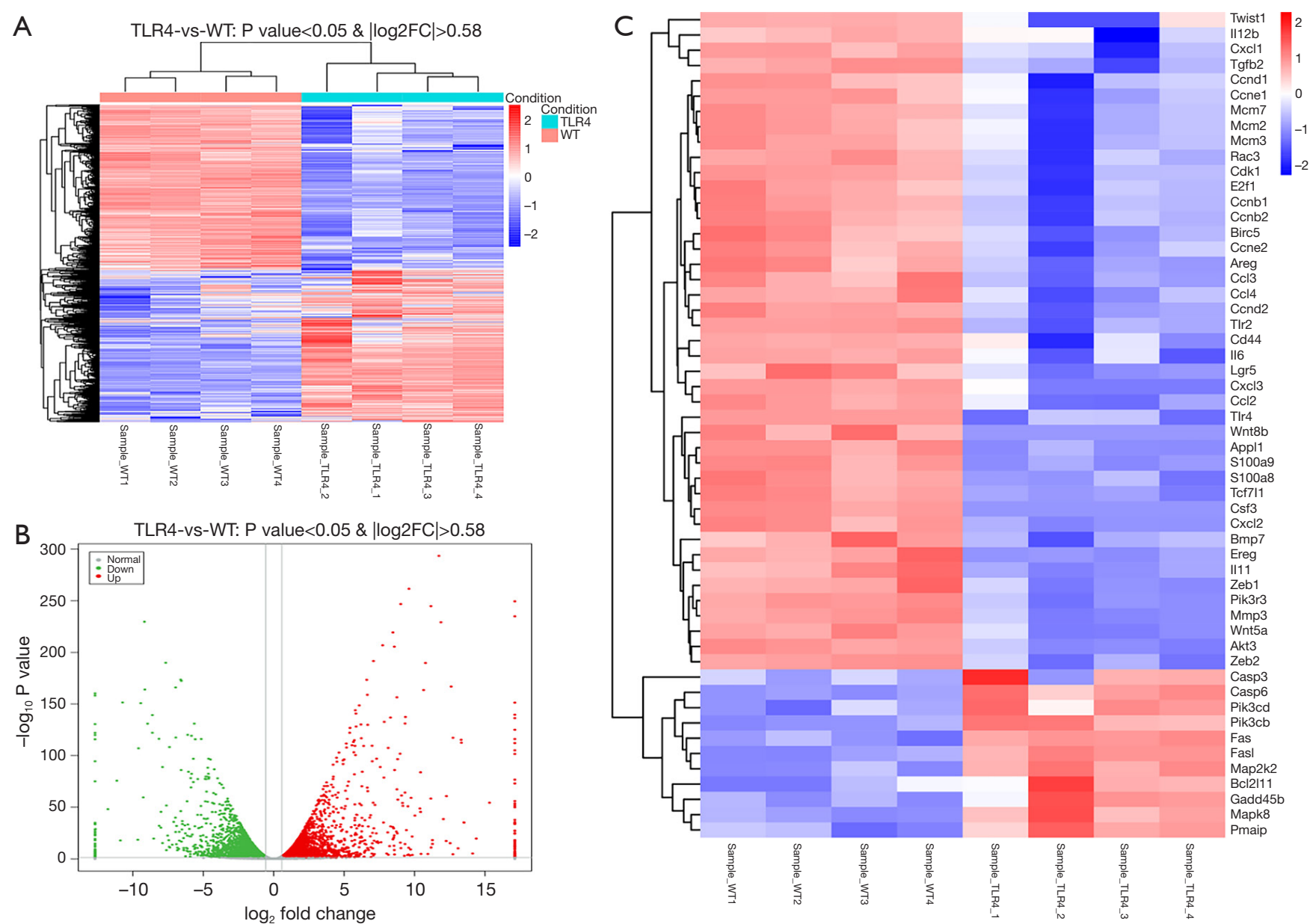

Figure 4 Differential genes expression between WT and TLR4-KO mice colons after dextran sulfate sodium (DSS) treatment were analyzed by high-throughput RNA-Seq. (A) Heat map of differential genes expression between WT and TLR4-KO mice colons after oral administration of $2.5 \%$ DSS for seven days. (B) Scatter plot of differently expressed genes in colon tissue. Each dot stands for a gene. Red and green color dots indicate an increase or decrease, respectively, of $>\log 2$ (fold change) of 0.58 and $\mathrm{P}<0.05$ in TLR4-KO group compared to the WT group. (C) Heat map of selected 54 genes with differential expression between WT and TLR4-KO mice after oral administration of $2.5 \%$ DSS for 7 days.

\section{TLR4-KO aggravates DSS-induced intestinal damage by attenuating cell cycle, cytokine-cytokine receptor interaction and TLR signaling patbways, and enhancing the apoptosis pathway}

We performed genes KEGG pathway enrichment analysis. Analysis of the KEGG database revealed differences in 319 pathways between the two groups following DSS treatment. Out of the total differential KEGG pathways between WT and TLR4-KO mice, we selected 15 signaling pathways that are closely related to DSS-induced colitis and performed KEGG enrichment. The four pathways with the highest enrichment scores were cell cycle $(\mathrm{P}<0.0001)$, cytokine- cytokine receptor interaction $(\mathrm{P}=0.0036)$, apoptosis $(\mathrm{P}=0.0429)$ and toll-like receptor signaling pathways $(\mathrm{P}=0.0615)$ (Figure 5). The expressions of important regulatory cyclins of the cell cycle pathway $(C y c B, C y c D$, $C y c E$, and $C D K 1$ ) were significantly lower, and $P 15$ and $P 21$ were significantly higher in the TLR4-KO gut tissue than in the WT control. Other important regulatory molecules in the cell cycle pathway, such as CDK4, CDK6 and P16, were not significantly different between WT control and TLR4-KO (Figure S1A). According to previous reports, down-regulated cyclins $(C y c B, C y c D, C y c E$ and $C D K 1)$ in the gut of TLR4-KO mice could promote the cell cycle pathway (19,20), while up-regulated P15 (21) and P21 (22) 


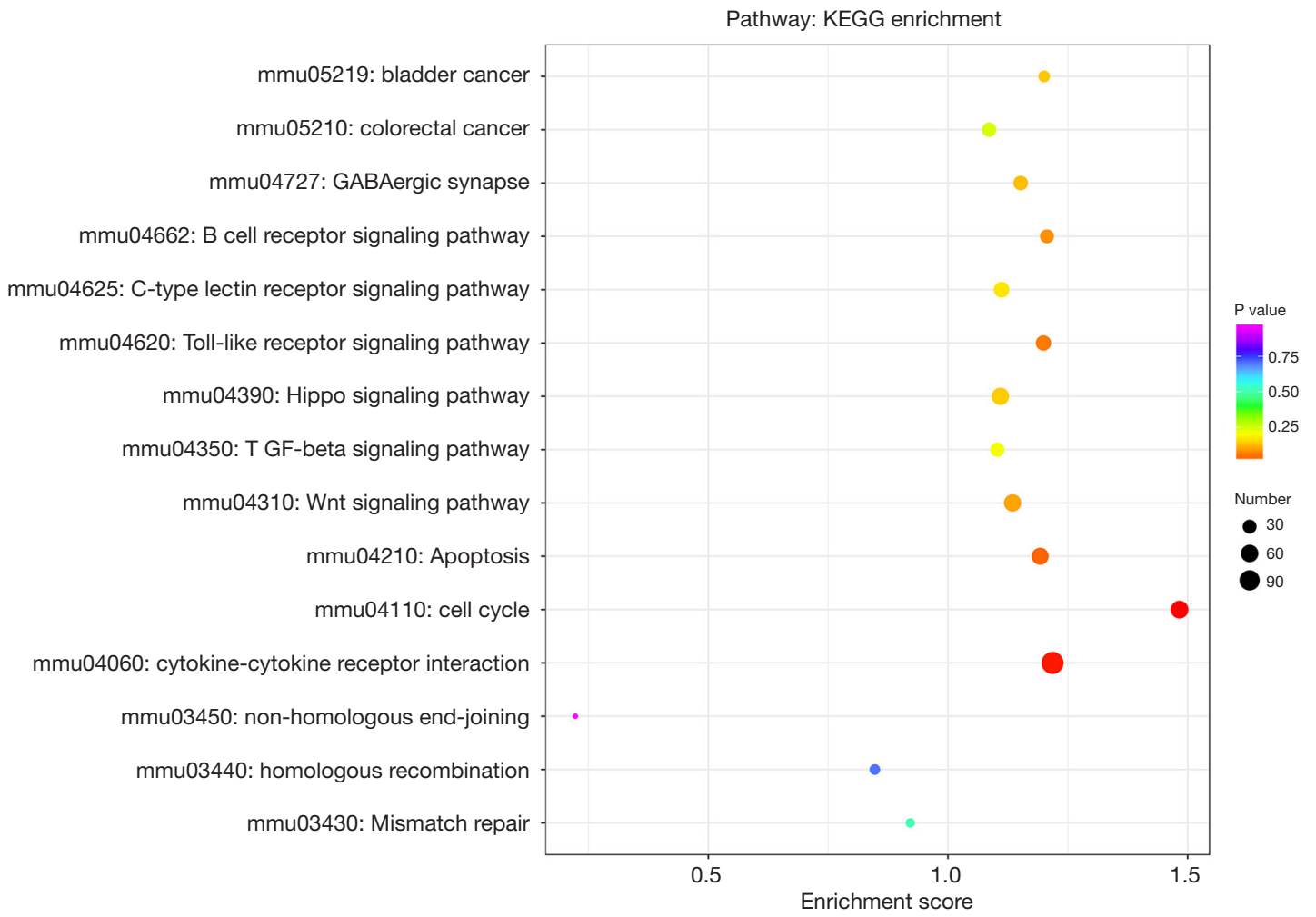

Figure 5 Fifteen signaling pathways related to dextran sulfate sodium (DSS)-induced colitis KEGG enrichment. Deletion of TLR4 activated cell cycle, cytokine-cytokine receptor interaction, and apoptosis signaling pathways, and enhanced DSS-induced intestinal damage.

in the gut of TLR4-KO mice could inhibit the cell cycle pathway. So, the cell cycle pathway was down-regulated in TLR4-KO mice following DSS treatment. In the cytokinecytokine receptor interaction pathway, expressions of the important regulatory factors $I L 11, I L 6, C C L 2, C S F 3$, and $T N F$ was significantly lower in TLR4-KO mice gut tissue compared to WT control (Figure S1B). All these regulatory factors play important roles in promoting the cytokinecytokine receptor interaction pathway $(23,24)$. It is thus clear that the cytokine-cytokine receptor interaction pathway was down-regulated in the guts of TLR4-KO mice following DSS treatment. Fas and Fas- $L$ are very important cytokines in the apoptosis signaling pathway, upregulation of which could significantly enhance the apoptosis signaling pathway (25). Expression of Fas and Fas-L significantly increased in the gut of TLR4-KO mice compared with WT control (Figure S2A). However, expression of $T N F-\alpha$ in the apoptosis signaling pathway significantly decreased in gut tissue of TLR4-KO mice compared to WT control. $T N F$ receptor signaling has an important role in repairing intestinal epithelium in acute intestinal injury (26)
(Figure S2A). These findings suggest that the apoptosis signaling pathway was generally up-regulated in TLR4-KO mice that were treated with DSS. The toll-like receptor signaling pathway showed a downward trend in the gut of TLR4-KO mice ( $\mathrm{P}=0.0615)$, however expression of TLR1, TLR2, TLR4, TLR5 and their downstream cytokines IL6, $I L 12$ and $T N F-\alpha$ significantly decreased in this pathway (Figure S2B). It can be concluded from all the above KEGG data that TLR4-KO might aggravate DSS-induced intestinal damage by attenuating the cell cycle, cytokine-cytokine receptor interaction, and toll-like receptor signaling pathways, and enhancing the apoptosis pathway.

\section{Twenty-five key genes screened by RNA-Seq and verified by $q R T-P C R$ and ELISA showed that IL-6, CCL2, and CSF3 are mediated by TLR4 in CT-26 cells}

qRT-PCR was performed for the 25 concerned genes, selected from all identified dysregulated differential genes. These 25 genes were significantly dysregulated in TLR4-KO mice colon as determined by RNA-Seq analysis, and were 
Table 2 Information on 25 differentially dysregulated genes is listed

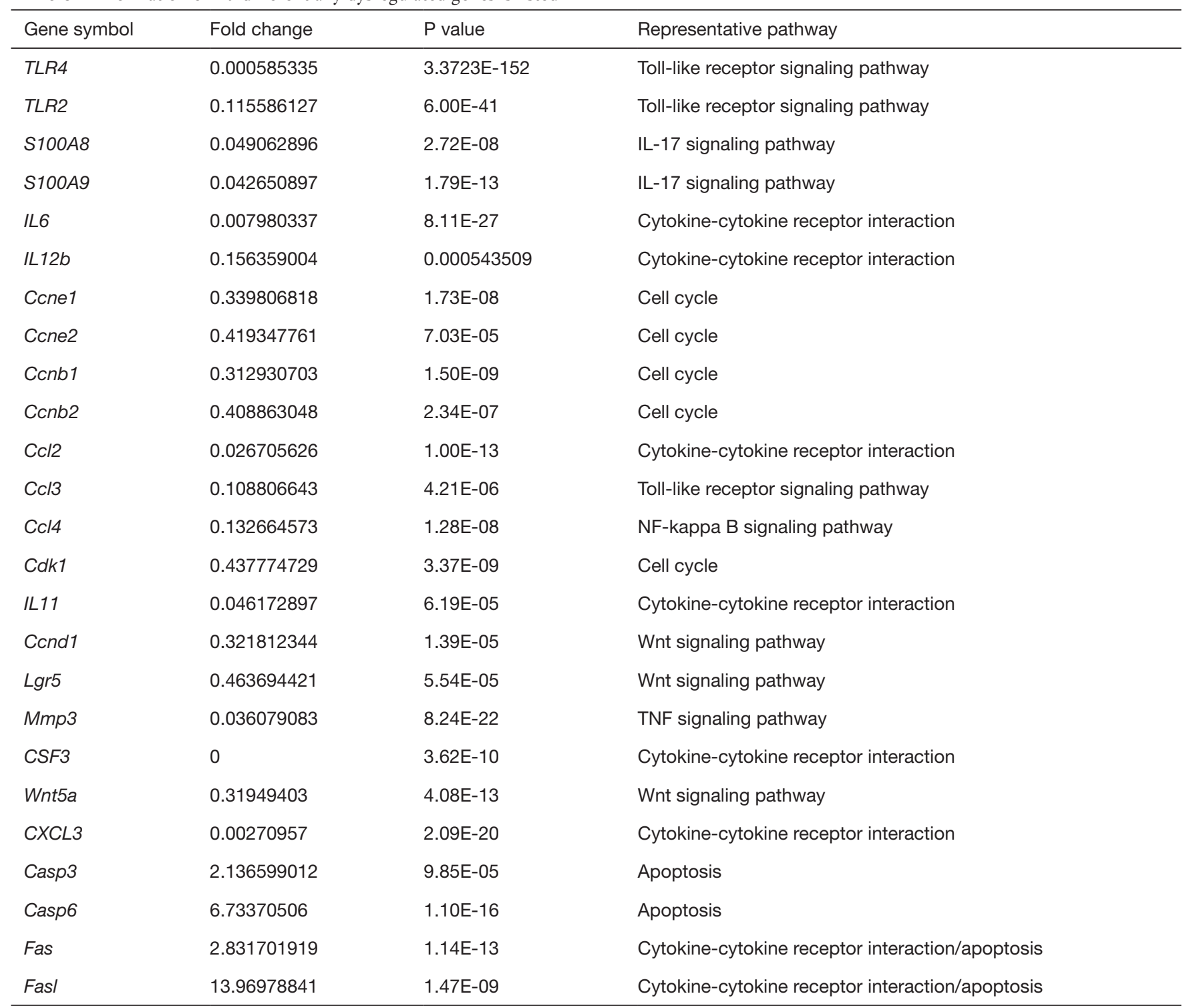

The related information presented include: gene symbol, fold change, $\mathrm{P}$ value, and representative pathway. Gene names written in green indicate that the genes were down-regulated in colons of TLR4-KO mice, when compared to the WT control. Gene names written in red indicate that the genes were up-regulated in colons of TLR4-KO mice, when compared to the WT control.

commonly used in our experimental system. The related information on these 25 differentially dysregulated genes is presented in Table 2. qRT-PCR results were consistent with the results of the RNA-Seq study, and, in comparison to the WT mice, expressions of TLR4, IL6, CCL2, and CSF3 were especially lower and Casp3, Casp6, Fas and Fasl were higher in the gut of TLR4-KO mice (Figure 6). ELISA was performed to measure expression levels of $I L-6, C C L 2$, and CSF 3 in the colon of WT and TLR4-KO mice. The results suggest that, compared with $W T$ mice, IL6, CCL2, and CSF3 were significantly down-regulated in TLR4-KO mice (Figure $7 A$ ). To explore how TLR4 signaling regulated the expression levels of IL6, CCL2, CSF3, Fas, and FasL, we performed qRT-PCR and ELISA in CT-26 cells treated with LPS. We found that the expression levels of IL6, CCL2, and $C S F 3$ significantly increased while the expression levels of Fas and FasL only slightly increased in CT-26 cells stimulated by LPS (Figure 7B,C). As far as Fas and FasL, the results were in disagreement with the results of the RNA-seq and qRT-PCR analyses that showed a significant increase 

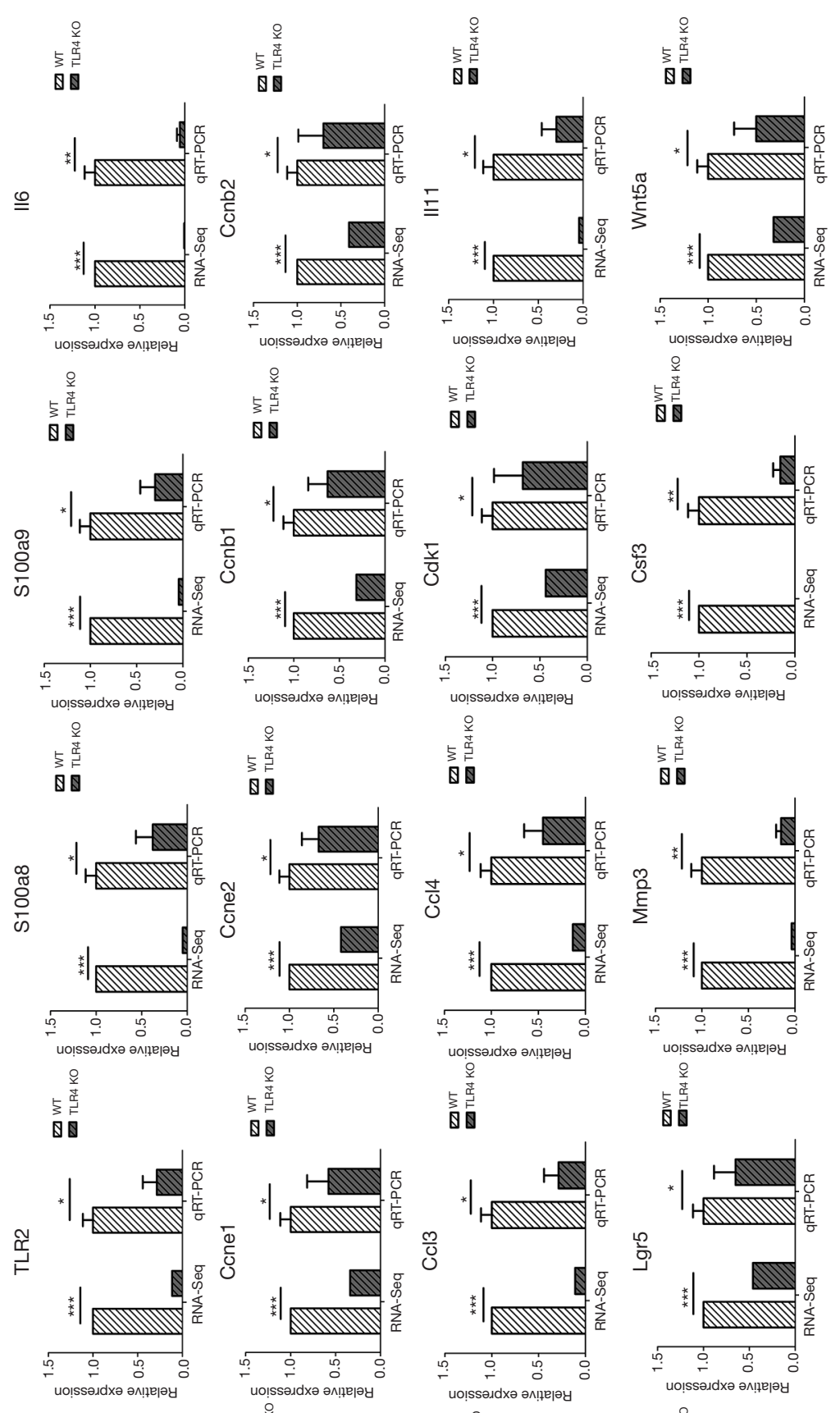

Q
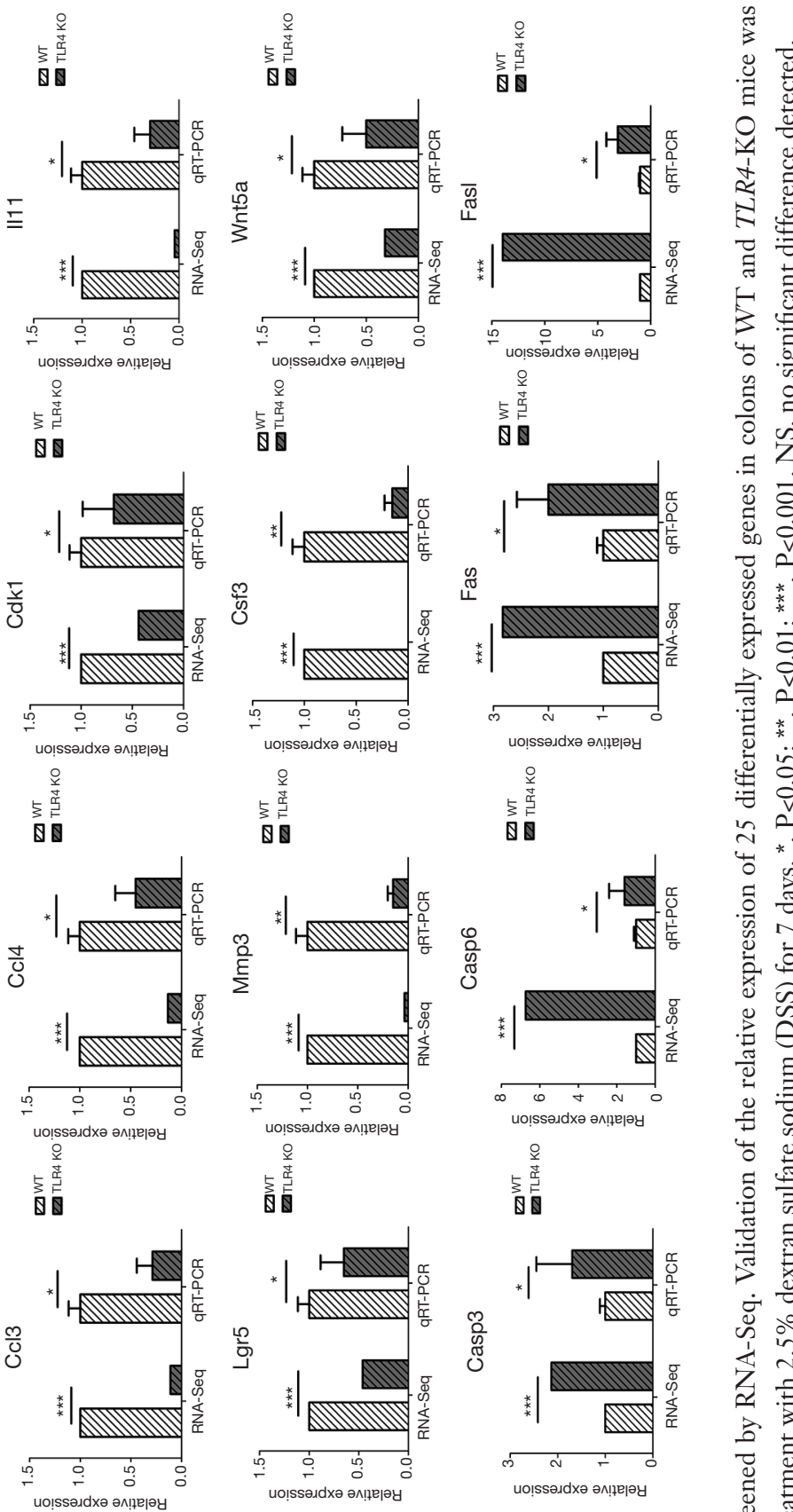

Z

ษे

के

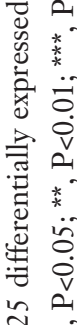

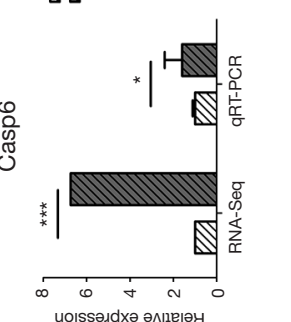

范

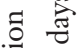

要

这

会

氖

吾目
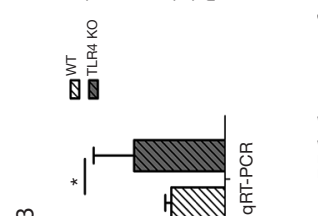

范壱

苟

일
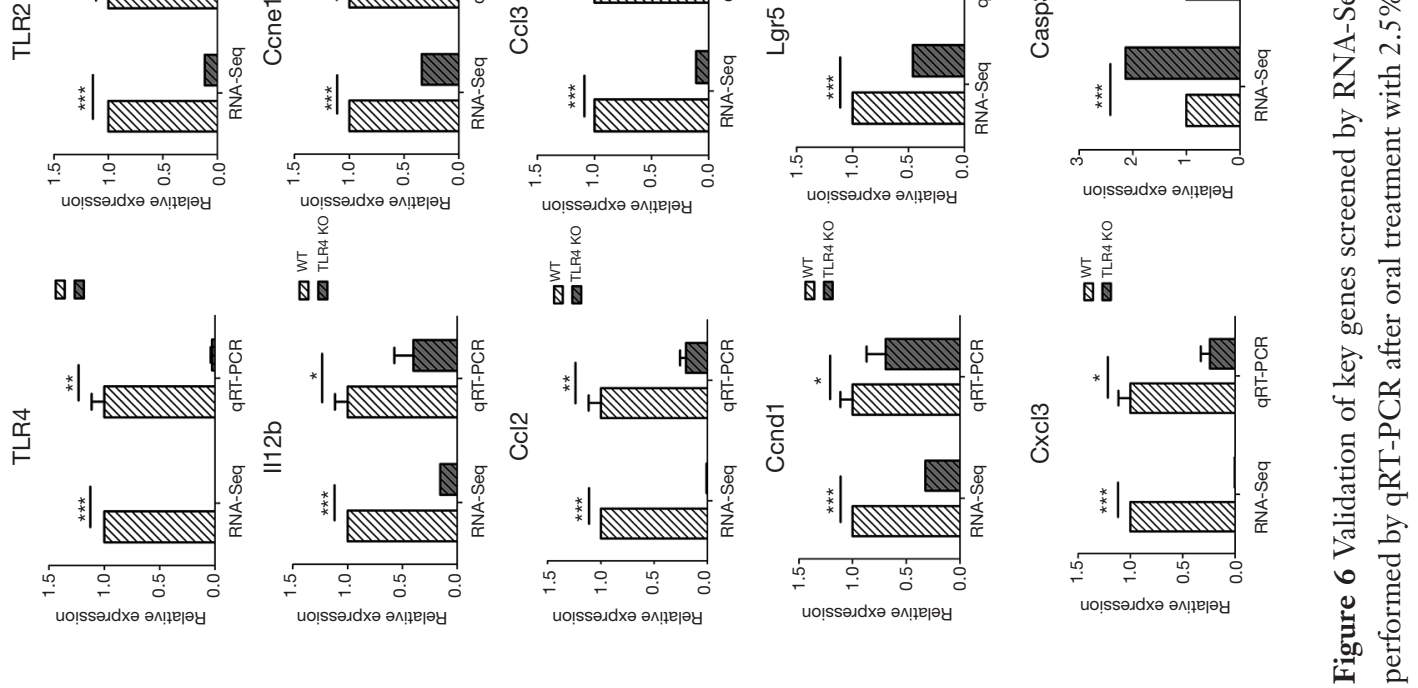
A

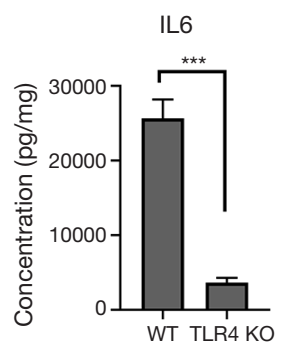

CCL2

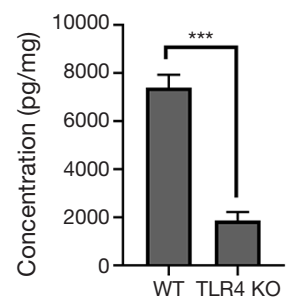

CSF3

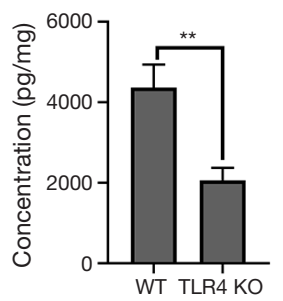

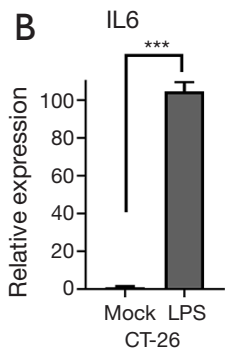
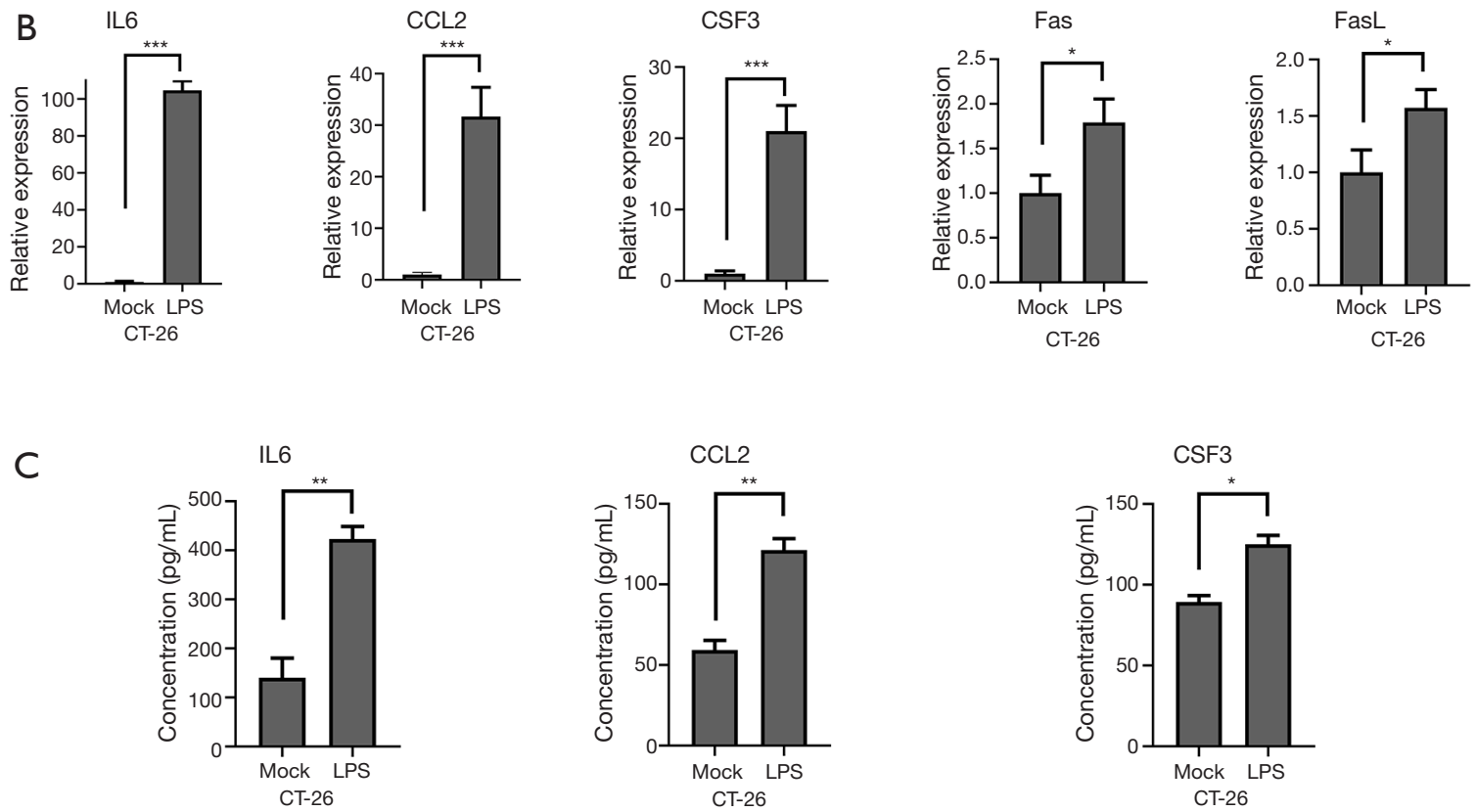

Figure 7 IL6 was related to TLR4 deficiency the most. The cytokines might be important in TLR4 signaling regulation of DSS injury, and that Fas might also be involved in the process, but if so, the effect is minimal. (A) ELISA validation for the concentration of IL6, CCL2, and CSF3 in colons of WT and TLR4-KO mice after treatment with 2.5\% dextran sulfate sodium (DSS) for seven days. (B) qRT-PCR evaluation of the expression levels of IL6, CCL2, CSF3, Fas, and FasL in the CT-26 cells, with and without LPS treatment (100 ng/mL). (C) ELISA validation for the concentration of IL6, CCL2, and CSF3 in CT-26 cells, with and without LPS treatment (100 ng/mL). *, P<0.05; **, $\mathrm{P}<0.01 ;{ }^{* * *}, \mathrm{P}<0.001$.

of Fas and FasL in the colon of TLR4-KO mice treated with DSS, because LPS has the effect of activating TLR4. These data suggested that TLR4 might play a repairing role in DSS-induced intestinal damage by up-regulating the cytokines IL6, CCL2, and CSF3. Fas and FasL promoted DSS-induced colon injury in the mice, but they might have little to do with TLR4 signaling.

\section{Functional study of the core dysregulated cytokines in intestinal cancer cell lines}

We next conducted a study on CT-26 cells (mouse intestinal cancer cell line), evaluating the functionality of the significantly dysregulated cytokines. CT-26 cells were treated with LPS (TLR4 agonist), IL6, IL11, CSF3, CCL2, S100A8, S100A9, MMP3, and Jo-2 (Fas agonistic monoclonal antibody) for $24 \mathrm{~h}$. The cells were then treated with $0.5 \%$ DSS. Cells viability was assessed after $24 \mathrm{~h}$, using the MTT assay. Results suggest that, compared with control, viability of DSS-treated CT-26 cells have decreased following treatment with Jo-2 and increased when treated with LPS, IL6, IL11, CSF3, CCL2, S100A8, S100A9, and MMP3. Notably, LPS, IL6, and CCL2 had a highly significant viability enhancement effect on DSS- 

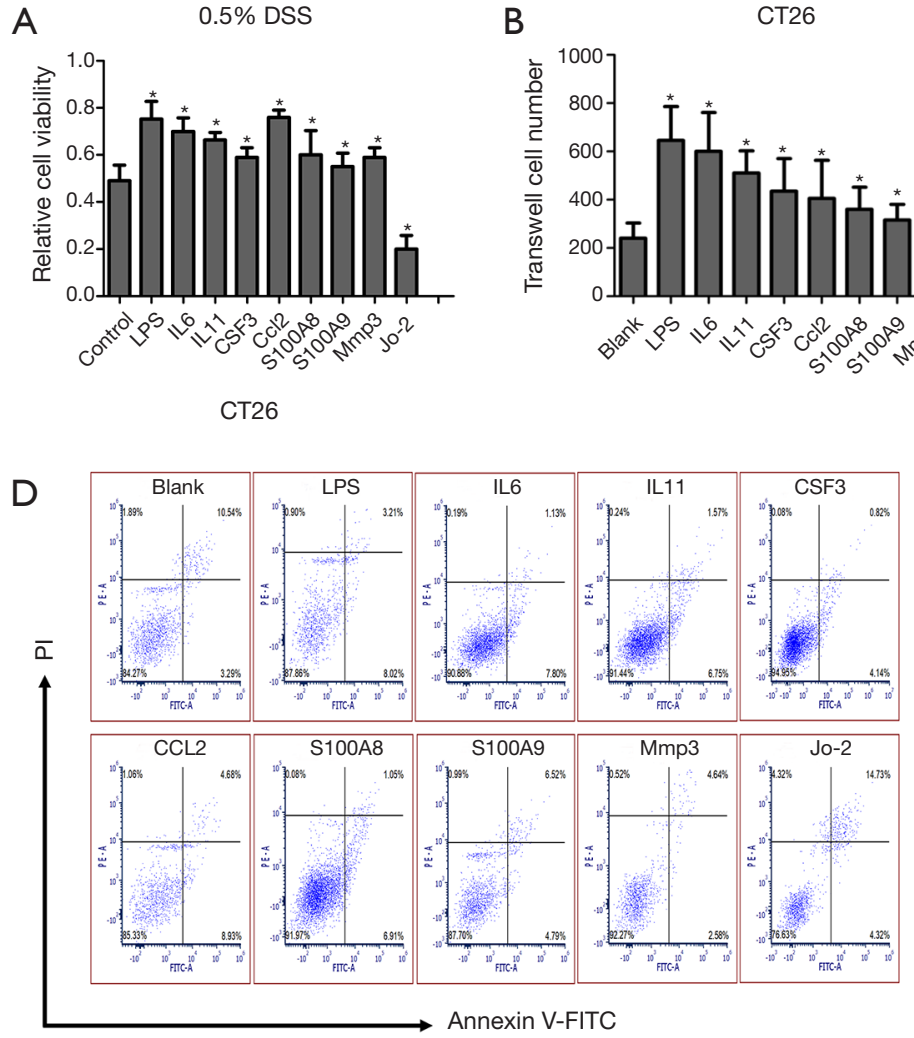
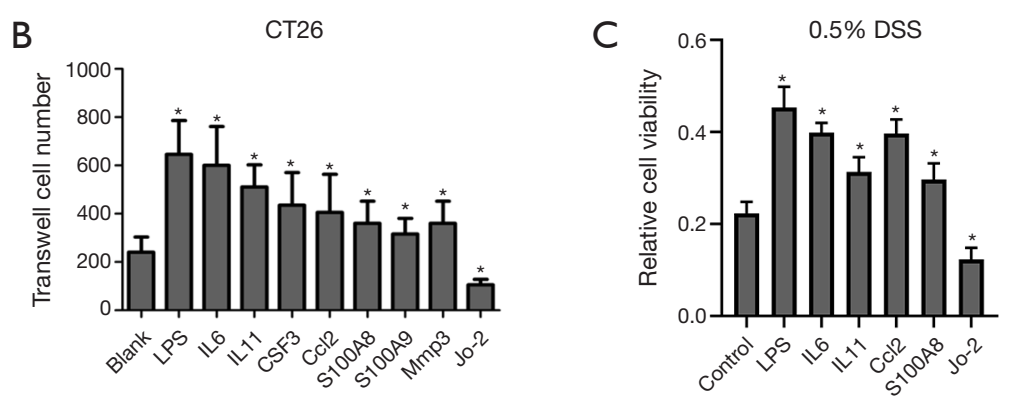

SW1116

$E$

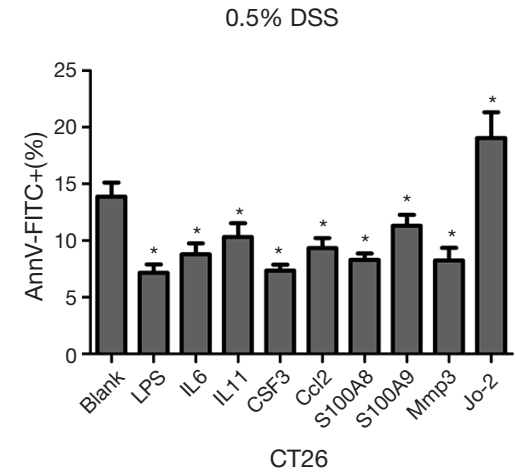

Figure 8 Functional study of the core dysregulated cytokines in CT-26 and SW1116 cells. (A) CT-26 cells were treated with $100 \mathrm{ng} / \mathrm{mL}$ LPS, IL6, IL11, CSF3, CCL2, S100A8/9, MMP3, and Jo-2 for $24 \mathrm{~h}$, after which they were treated with $0.5 \%$ dextran sulfate sodium (DSS). Cells viability was evaluated after $24 \mathrm{~h}$, using the MTT assay. (B) Migration assessment of CT-26 cells treated with LPS, IL6, IL11, CSF3, CCL2, S100A8/9, MMP3, and Jo-2. (C) SW1116 cells were treated with $100 \mathrm{ng} / \mathrm{mL}$ LPS, IL6, IL11, CCL2, S100A8, and Jo-2 for $24 \mathrm{~h}$, after which they were treated with $0.5 \%$ DSS. Cells viability was evaluated after $24 \mathrm{~h}$, using the MTT assay. (D) CT-26 cells were treated with $100 \mathrm{ng} / \mathrm{mL}$ LPS, IL6, IL11, CSF3, CCL2, S100A8/9, MMP3, and Jo-2 for $24 \mathrm{~h}$, after which they were treated with $0.5 \%$ DSS. Apoptosis rate was assessed by flow cytometry after $24 \mathrm{~h}$, using Annexin V/PI dual staining. (E) Statistical analysis of Annexin V-FITC-positive DSStreated CT-26 cells. *, $\mathrm{P}<0.05$.

treated CT-26 cells (Figure 8A). We also performed the transwell migratory assay to study the effect of LPS, IL6, IL11, CSF3, CCL2, S100A8, S100A9, MMP3 and Jo-2 on intestinal cells migration. Consistent with previous experimental results, when compared with the blank control, LPS, IL6, IL11, CSF3, CCL2, S100A8, S100A9 and $M M P 3$ promoted CT-26 cells' migration and Jo-2 inhabited it (Figure 8B). Additionally, SW1116 cells (human colorectal cancer cell line) were treated with LPS, IL6, IL11, CCL2, S100A8, and Jo-2 for $24 \mathrm{~h}$, after which the cells were treated with $0.5 \%$ DSS. Cells viability was assessed after $24 \mathrm{~h}$, using the MTT assay. Results suggest that, compared to the control, viability of DSS-treated SW1116 cells has decreased following treatment with Jo2, and increased when treated with LPS, IL6, IL11, CCL2,
S100A8. The increase was especially notable when the cells were treated with LPS, IL6, and CCL2 (Figure 8C). Apoptosis rate of cytokines-treated CT-26 cells that were exposed to $0.5 \%$ DSS was then examined by Annexin V/ PI dual staining. It was found that, compared to the blank control, apoptosis rates of LPS, IL6, IL11, CSF3, CCL2, S100A8, S100A9, and MMP3-treated CT-26 cells have decreased and the apoptosis rate of Jo-2-treated CT26 cells has increased after $0.5 \%$ DSS administration (Figure 8D,E).

\section{Discussion}

TLRs are sensors for pathogen-associated molecular patterns (PAMPs) and play important roles in immune 


\section{Page 14 of 16}

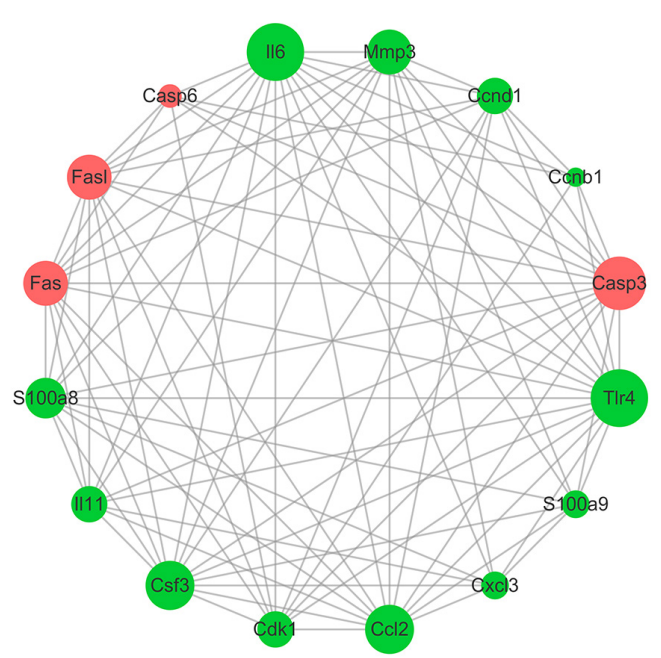

Figure 9 Network interaction map of genes in the guts of TLR4$\mathrm{KO}$ mice.

responses (27). TLR4 is a pattern recognition receptor (PRR) found in IEC that recognize microbial molecules. Research has shown that after TLR4 combines with LPS, it triggers signaling cascades, mediated by MyD88, to activate the transcription factor $N F-\kappa B$ and, eventually, leads to inflammatory response (28). However, in our work, we found that LPS and TLR4-KO aggravated intestinal damage in DSS-induced colitis in mouse model.

In order to study the key genes involved in TLR4-KO prompting DSS-induced intestinal injury, we used highthroughput RNA sequencing to screen for differentially expressed genes in the gut, comparing between WT and TLR4-KO mice. qRT-PCR was performed to verify our singled-out genes and results were consistent with the RNA-Seq data. Previous studies (26) have reported that the inflammatory cytokine IL6 could protect IEC through the activation of STAT3. TNF receptor signaling was also shown to have an important role in repairing intestinal epithelium in acute intestinal injury (26). The repair genes, Ccnb1, Ccnd1, CSF3 and CCL2, are involve in mediating multiple pathways to repair intestinal epithelium in DSSinduced intestinal injury $(29,30)$. Combined with the KEGG enrichment data, our study shows that IL6, CCL2, CSF3, IL11, Ccnb1, Ccnd1 and TNF- $\alpha$ have significantly decrease and Fas and Fas-L have increase in DSS-induced colitis related pathways. The expression levels of IL 6 , CCL2, CSF3, Fas and FasL have all increased in CT-26 cells stimulated by LPS. Fas and FasL have also increased in the colon of TLR4-KO mice. These data suggest that TLR4$\mathrm{KO}$ aggravates DSS-induced colon injury through downregulation of the cytokines IL6, CCL2, and CSF3. Fas and Fas $L$ promoted DSS-induced colon injury in mice, but this might have little to do with TLR4 signaling. Additionally, we confirmed that, when compared to the blank control, treatment with LPS (TLR4 agonist), IL6, IL11, CSF3, CCL2, S100A8, S100A9 and Mmp3 enhanced CT-26 cells migration, and treatment with Jo-2 (Fas agonistic monoclonal antibody) inhabited it. LPS, IL6, IL11, CSF3, CCL2, S100A8, S100A9 and Mmp3 have also improved cell viability and reduced apoptosis rate in DSS-treated CT-26 cells. Jo-2, so we found, plays the opposite role. This indicates that promotion of cytokines activity, such as IL6 and CCL2, or inhibition Fas activity could effectively improve the repair of intestinal epithelial damage. Based on these results, we built the genes network interaction map (Figures 9 and S3).

\section{Acknowledgments}

Funding: This work was supported by the National Basic Research Program of China, 973 Program (grant number 2015CB554000) and the National Natural Science Foundation of China (grants numbers 81573092, 81872046 $\& 81872559)$.

\section{Footnote}

Conflicts of Interest: The authors have no conflicts of interest to declare.

Ethical Statement: The authors are accountable for all aspects of the work in ensuring that questions related to the accuracy or integrity of any part of the work are appropriately investigated and resolved. This study was approved by an institutional ethics committee and followed the tenets of the Declaration of Helsinki.

\section{References}

1. Uniken Venema WT, Voskuil MD, Dijkstra G, et al. The genetic background of inflammatory bowel disease: from correlation to causality. J Pathol 2017;241:146-58.

2. Ni J, Wu GD, Albenberg L, et al. Gut microbiota and IBD: causation or correlation? Nat Rev Gastroenterol Hepatol 2017;14:573-84.

3. Nosratababadi R, Bagheri V, Zare-Bidaki M, et al. Toll 
like receptor 4: an important molecule in recognition and induction of appropriate immune responses against Chlamydia infection. Comp Immunol Microbiol Infect Dis 2017;51:27-33.

4. Kannaki TR, Priyanka E, Reddy MR. Co-administration of toll-like receptor (TLR)-3 and 4 ligands augments immune response to Newcastle disease virus (NDV) vaccine in chicken. Vet Res Commun 2019;43:225-30.

5. Liu J, Chen Y, Liu D, et al. Ectopic expression of SIGIRR in the colon ameliorates colitis in mice by downregulating TLR4/NF-kappaB overactivation. Immunol Lett 2017;183:52-61.

6. Shi YJ, Gong H, Zhao Q, et al. Critical Role of TollLike Receptor 4 (TLR4) in Dextran Sulfate Sodium (DSS)-Induced Intestinal Injury and Repair. Toxicol Lett 2019;315:23-30.

7. Cario E, Rosenberg IM, Brandwein SL, et al. Lipopolysaccharide Activates Distinct Signaling Pathways in Intestinal Epithelial Cell Lines Expressing Toll-Like Receptors. J Immunol 2000;164:966-72.

8. Rakoff-Nahoum S, Paglino J, Eslami-Varzaneh F, et al. Recognition of commensal microflora by toll-like receptors is required for intestinal homeostasis. Cell 2004;118:229-41.

9. Hoffmann M, Schwertassek U, Seydel A, et al. A refined and translationally relevant model of chronic DSS colitis in BALB/c mice. Lab Anim 2018;52:240-52.

10. Chung YW, Choi JH, Oh TY, et al. Lactobacillus casei prevents the development of dextran sulphate sodiuminduced colitis in Toll-like receptor 4 mutant mice. Clin Exp Immunol 2008;151:182-9.

11. Iatropoulos MJ, Williams GM. Proliferation markers. Exp Toxicol Pathol 1996;48:175-81.

12. Trapnell C, Williams BA, Pertea G, et al. Transcript assembly and quantification by RNA-Seq reveals unannotated transcripts and isoform switching during cell differentiation. Nat Biotechnol 2010;28:511-5.

13. Mao W, Huang X, Wang L, et al. Circular RNA hsa_ circ_0068871 regulates FGFR3 expression and activates STAT3 by targeting miR-181a-5p to promote bladder cancer progression. J Exp Clin Cancer Res 2019;38:169.

14. Chervoneva I, Freydin B, Hyslop T, et al. Modeling qRTPCR dynamics with application to cancer biomarker quantification. Stat Methods Med Res 2018;27:2581-95.

15. Chin AC, Flynn AN, Fedwick JP, et al. The role of caspase- 3 in lipopolysaccharide-mediated disruption of intestinal epithelial tight junctions. Can J Physiol Pharmacol 2006;84:1043-50.
16. Tischlerova V, Kello M, Budovska M, et al. Indole phytoalexin derivatives induce mitochondrial-mediated apoptosis in human colorectal carcinoma cells. World J Gastroenterol 2017;23:4341-53.

17. Liu C, Lin J, Zhao L, et al. Gamma-ray irradiation impairs dendritic cell migration to CCL19 by down-regulation of CCR7 and induction of cell apoptosis. Int J Biol Sci 2011;7:168-79.

18. Cochet F, Peri F. The Role of Carbohydrates in the Lipopolysaccharide (LPS)/Toll-Like Receptor 4 (TLR4) Signalling. Int J Mol Sci 2017;18. doi: 10.3390/ ijms18112318.

19. Lim S, Kaldis P. Cdks, cyclins and CKIs: roles beyond cell cycle regulation. Development 2013;140:3079-93.

20. Roy A, Banerjee S. p27 and leukemia: cell cycle and beyond. J Cell Physiol 2015;230:504-9.

21. Patsoukis N, Brown J, Petkova V, et al. Selective effects of PD-1 on Akt and Ras pathways regulate molecular components of the cell cycle and inhibit $\mathrm{T}$ cell proliferation. Sci Signal 2012;5:ra46.

22. Karimian A, Ahmadi Y, Yousefi B. Multiple functions of p21 in cell cycle, apoptosis and transcriptional regulation after DNA damage. DNA Repair (Amst) 2016;42:63-71.

23. Lin P, Guo YN, Shi L, et al. Development of a prognostic index based on an immunogenomic landscape analysis of papillary thyroid cancer. Aging (Albany NY) 2019;11:480-500.

24. Zhou $W$, Yin M, Cui H, et al. Identification of potential therapeutic target genes and mechanisms in non-smallcell lung carcinoma in non-smoking women based on bioinformatics analysis. Eur Rev Med Pharmacol Sci 2015;19:3375-84.

25. He $X, W u$ J, Yuan L, et al. Lead induces apoptosis in mouse TM3 Leydig cells through the Fas/FasL death receptor pathway. Environ Toxicol Pharmacol 2017;56:99-105.

26. Nguyen PM, Putoczki TL, Ernst M. STAT3-Activating Cytokines: A Therapeutic Opportunity for Inflammatory Bowel Disease? J Interferon Cytokine Res 2015;35:340-50.

27. Broz P, Ohlson MB, Monack DM. Innate immune response to Salmonella typhimurium, a model enteric pathogen. Gut Microbes 2012;3:62-70.

28. Siddique I, Khan I. Mechanism of regulation of $\mathrm{Na}-\mathrm{H}$ exchanger in inflammatory bowel disease: role of TLR-4 signaling mechanism. Dig Dis Sci 2011;56:1656-62.

29. Farooq SM, Hou Y, Li H, et al. Disruption of GPR35 Exacerbates Dextran Sulfate Sodium-Induced Colitis in Mice. Dig Dis Sci 2018;63:2910-22. 
Page 16 of 16

30. Stronati L, Palone F, Negroni A, et al. Dipotassium Glycyrrhizate Improves Intestinal Mucosal Healing by Modulating Extracellular Matrix Remodeling Genes and

Cite this article as: Shi YJ, Hu SJ, Zhao QQ, Liu XS, Liu C, Wang H. Toll-like receptor 4 (TLR4) deficiency aggravates dextran sulfate sodium (DSS)-induced intestinal injury by down-regulating IL6, CCL2 and CSF3. Ann Transl Med 2019;7(23):713. doi: 10.21037/atm.2019.12.28
Shi et al. TLR4-KO aggravates colitis by decreasing IL6, CCL2, CSF3

Restoring Epithelial Barrier Functions. Front Immunol 2019;10:939. 

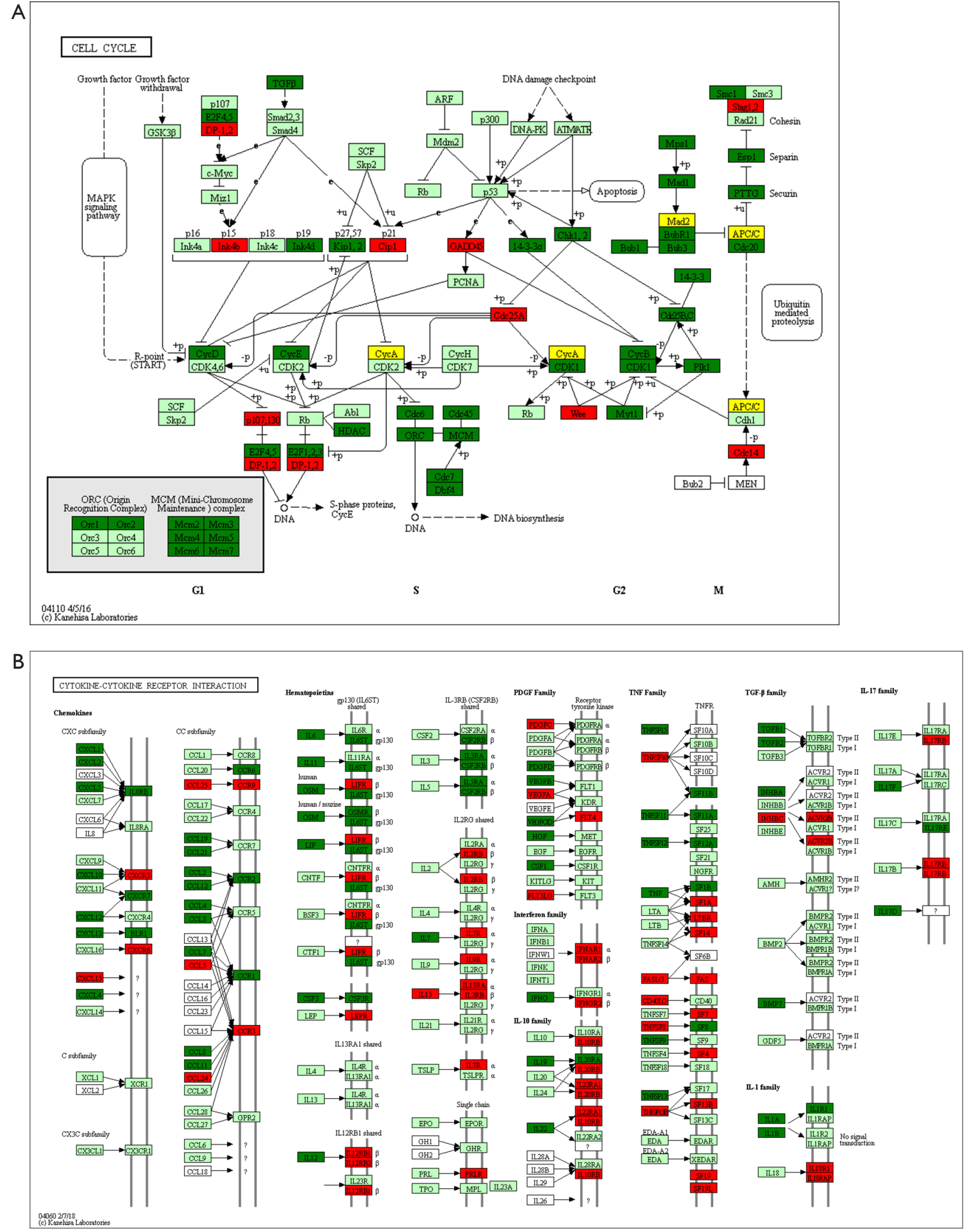

Figure S1 Toll-like receptor 4 (TLR4)-KO aggravates dextran sulfate sodium (DSS)-induced intestinal damage by attenuating cell cycle and cytokine-cytokine receptor interaction. (A) Map of cell cycle signaling pathway; (B) map of cytokine-cytokine receptor interaction signaling pathway. 

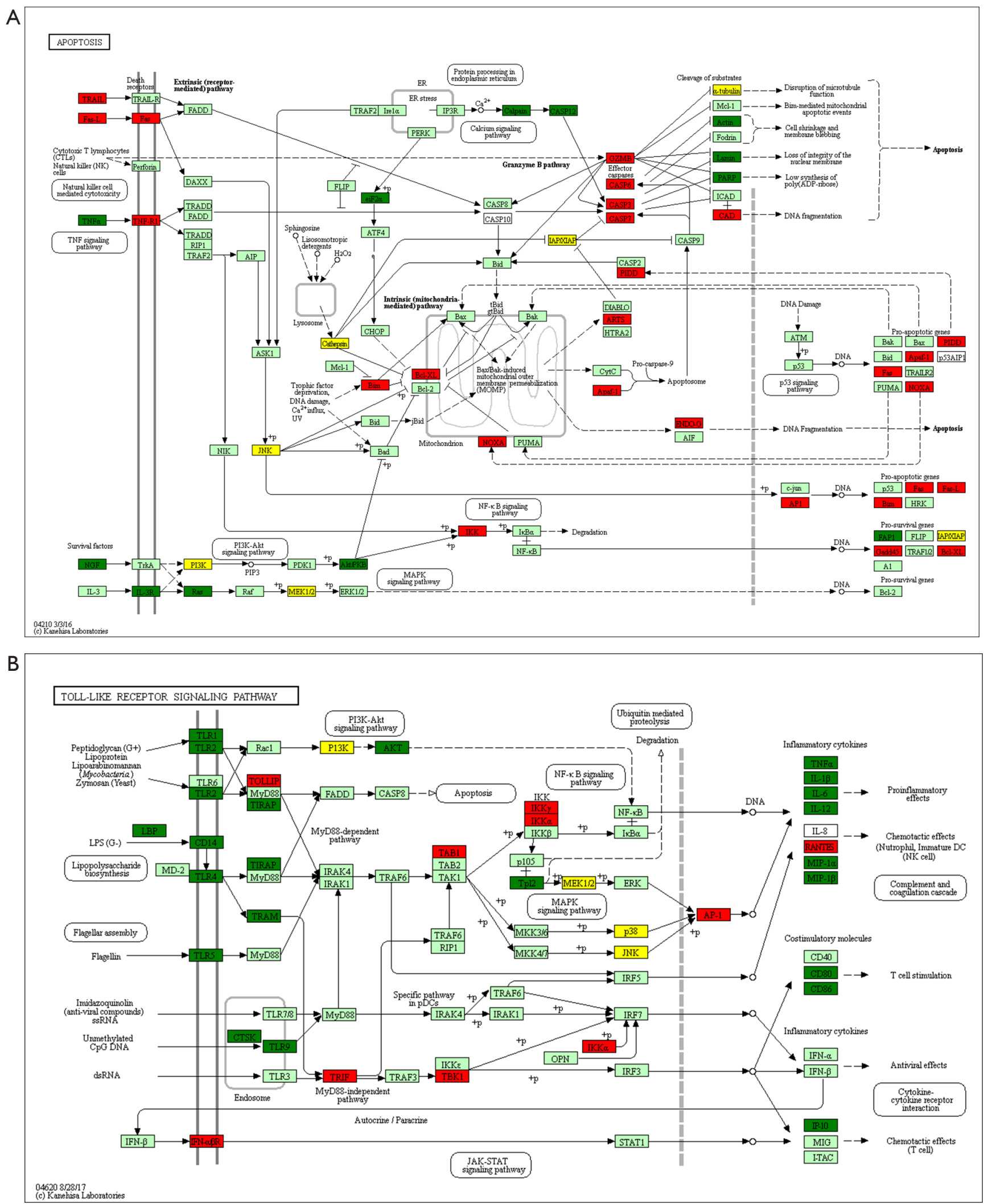

Figure S2 Toll-like receptor 4 (TLR4)-KO aggravates dextran sulfate sodium (DSS)-induced intestinal damage by attenuating TLR signaling pathways and enhancing the apoptosis pathway. (A) Map of apoptosis signaling pathway; (B) map of Toll-like receptor signaling pathway. 


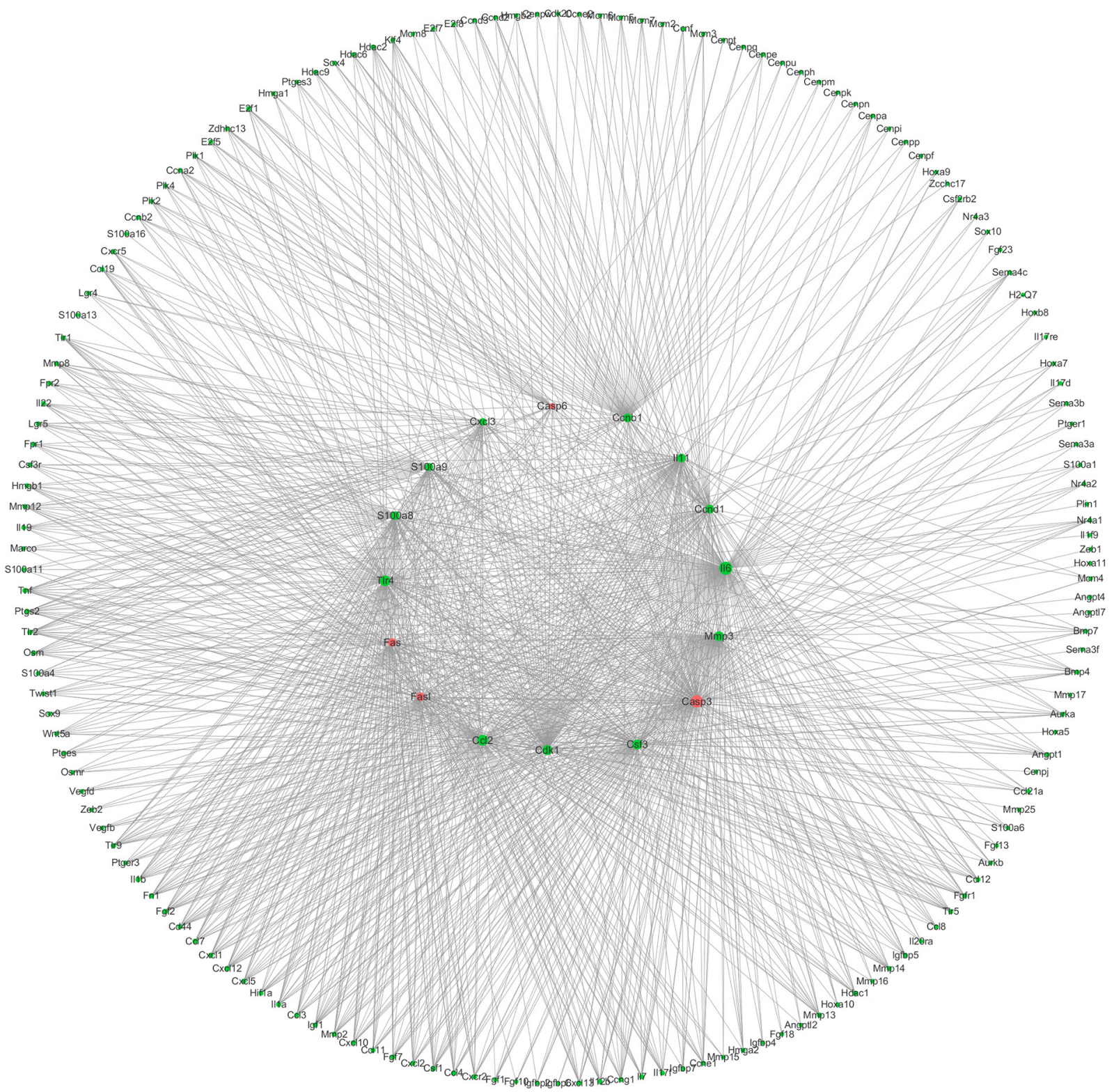

Figure S3 Network interaction map of genes in the guts of Toll-like receptor 4 (TLR4)-KO mice, and their core downstream factors, aggravating dextran sulfate sodium (DSS)-induced colitis. 